TRANSACTIONS OF THE

AMERICAN MATHEMATICAL SOCIETY

Volume 360, Number 6, June 2008, Pages 2923-2940

S 0002-9947(07)04394-2

Article electronically published on December 11, 2007

\title{
GRADED LEVEL ZERO INTEGRABLE REPRESENTATIONS OF AFFINE LIE ALGEBRAS
}

\author{
VYJAYANTHI CHARI AND JACOB GREENSTEIN
}

\begin{abstract}
We study the structure of the category of integrable level zero representations with finite dimensional weight spaces of affine Lie algebras. We show that this category possesses a weaker version of the finite length property, namely that an indecomposable object has finitely many simple constituents which are non-trivial as modules over the corresponding loop algebra. Moreover, any object in this category is a direct sum of indecomposables only finitely many of which are non-trivial. We obtain a parametrization of blocks in this category.
\end{abstract}

\section{INTRODUCTION}

In this paper, we continue the study $([2,3,7])$ of the category $\mathcal{I}^{\text {fin }}$ of integrable level zero representations with finite-dimensional weight spaces of affine Lie algebras. The category of integrable representations with finite-dimensional weight spaces and of non-zero level is semi-simple $([16])$, and the simple objects are the highest weight modules. In contrast, it is easy to see that the category $\mathcal{I}^{\text {fin }}$ is not semi-simple. For instance, the derived Lie algebra of the affine algebra itself provides an example of a non-simple indecomposable object in that category. The simple objects in $\mathcal{I}^{\text {fin }}$ were classified in $[2,7]$ but not much else is known about the structure of the category.

The category $\mathcal{I}^{\text {fin }}$ can be regarded as the graded version of the category $\mathcal{F}$ of finite-dimensional representations of the corresponding loop algebra. The structure of $\mathcal{F}$ has been studied extensively in both the quantum and classical cases in recent years $([1,5,6,9,14])$. There is a natural functor $L$ from $\mathcal{F}$ to $\mathcal{I}^{\text {fin }}$ which we study in this paper (Section 5 ) and which has many nice properties. But there are important properties that fail, for example in general the functor $L$ only maps irreducible objects to completely reducible objects. In particular, the trivial representation in $\mathcal{F}$ is mapped to an infinite direct sum of one-dimensional representations.

The latter phenomenon is the main source of difficulty and makes the study of the category $\mathcal{I}^{\text {fin }}$ interesting in its own right, since the results and proofs sometimes require substantial modifications from the ones for $\mathcal{F}$. It is immediate from the preceding comments that objects in the category $\mathcal{I}^{\text {fin }}$ are not always of finite length. However, in Section 4 of this paper, we show that a weaker version of the finite length property holds, namely that the number of non-trivial constituents of an indecomposable module is finite. Moreover, each indecomposable module admits

Received by the editors February 23, 2006.

2000 Mathematics Subject Classification. Primary 17B67.

This work was partially supported by the NSF grant DMS-0500751. 
an analogue of a composition series which we call a pseudo-Jordan-Hölder series. Such a series is unique up to a natural equivalence. As a result we are able to conclude (Theorem 1) that any object in $\mathcal{I}^{\text {fin }}$ is a direct sum of indecomposable modules. Moreover, only finitely many of the indecomposable summands are nontrivial modules for the corresponding loop algebra. This, in particular, implies that $\mathcal{I}^{\text {fin }}$ has a block decomposition. The methods used in this part of the paper rely only on facts which remain valid for quantum affine algebras.

In Section 6 we obtain a parametrization for the blocks and describe the blocks explicitly (Theorem 2). The blocks in $\mathcal{I}^{\text {fin }}$ are parametrized by the orbits for the natural action of the group $\mathbf{C}^{\times}$on the set $\Xi$ of finitely supported functions from $\mathbf{C}^{\times}$to the quotient group of the weight lattice of the underlying simple finitedimensional Lie algebra $\mathfrak{g}$ by its root lattice. It was proved in [5] that $\Xi$ parametrizes blocks in the category $\mathcal{F}$. To prove the result for $\mathcal{I}^{\text {fin }}$ we use the functor $L$ defined and studied in Section 5. One of the tools used crucially in [5] was the notion of the finite-dimensional Weyl module introduced and studied in [8] (in the quantum case they appear in $[12,13])$. One can define in a similar way the notion of the graded Weyl module, which is an object in $\mathcal{I}^{\text {fin }}$ and has the usual universal properties. A natural question is then whether the functor $L$ maps Weyl modules in $\mathcal{F}$ to graded Weyl modules or at least to direct sums of graded Weyl modules. This question turns out to be rather difficult since it is equivalent to proving the conjecture of [8] on the dimension of the Weyl modules in $\mathcal{F}$. This conjecture was established in [8] for the affine algebra whose underlying simple Lie algebra $\mathfrak{g}$ is isomorphic to $\mathfrak{s l}_{2}$. In other cases, as Hiraku Nakajima has pointed out to us recently, the dimension conjecture can be deduced as follows. The results of $[12,13]$ imply that the Weyl modules are specializations (the $q=1$ limit) of certain finite-dimensional quotients of the extremal modules for the quantum affine algebra. Then it follows from the results in [1] and $[14,15]$ that these quotients (and hence their specializations) have the correct dimension. Other approaches to proving this conjecture, which do not rely on the quantum case, have been studied recently: in [4] for the case of $\mathfrak{g}$ isomorphic to $\mathfrak{s l}_{r}, r \geq 3$ and in [10] for any simply-laced $\mathfrak{g}$.

\section{Preliminaries}

Throughout the paper, $\mathbf{Z}$ (respectively, $\mathbf{Z}_{+}, \mathbf{N}$ ) will denote the set of integers (respectively, non-negative, positive integers).

2.1. Let $\mathfrak{g}$ be a complex finite-dimensional simple Lie algebra and $\mathfrak{h}$ a Cartan subalgebra of $\mathfrak{g}$. Set $I=\{1, \ldots, \operatorname{dim} \mathfrak{h}\}$ and let $\left\{\alpha_{i}: i \in I\right\}$ (respectively, $\left\{\varpi_{i}: i \in I\right\}$ ) be a set of simple roots (respectively, fundamental weights) of $\mathfrak{g}$ with respect to $\mathfrak{h}$, and $R^{+}$(respectively, $Q, P$ ) be the corresponding set of positive roots (respectively the root lattice, the weight lattice). Let $Q^{+}, P^{+}$be the $\mathbf{Z}_{+}$-span of the simple roots and fundamental weights respectively. It is convenient to set $\varpi_{0}=0$. Let $\geq$ be the standard partial order on $P$ defined by: $\lambda \geq \mu$ if $\lambda-\mu \in Q^{+}$. Let $\theta \in R^{+}$be the highest root and if $\mathfrak{g}$ is not simply laced denote by $\theta_{s}$ the highest short root. Denote by $W$ the Weyl group of $\mathfrak{g}$.

Given $\alpha \in R$, let $\mathfrak{g}_{\alpha}$ be the corresponding root space. For $\alpha \in R^{+}$, fix non-zero elements $x_{\alpha}^{ \pm} \in \mathfrak{g}_{ \pm \alpha}$ and $\alpha^{\vee} \in \mathfrak{h}$, such that

$$
\left[\alpha^{\vee}, x_{\alpha}^{ \pm}\right]= \pm 2 x_{\alpha}^{ \pm}, \quad\left[x_{\alpha}^{+}, x_{\alpha}^{-}\right]=\alpha^{\vee}
$$


and write

$$
\mathfrak{n}^{ \pm}=\bigoplus_{\alpha \in R^{+}} \mathfrak{g}_{ \pm \alpha}=\bigoplus_{\alpha \in R^{+}} \mathbf{C} x_{ \pm \alpha}
$$

It is well-known that $\Gamma=P / Q$ is a finite abelian group. For any $0 \neq \gamma \in \Gamma$ fix the unique minimal representative $0 \neq \varpi_{\gamma} \in P^{+}$, i.e. if $\lambda \in P^{+}$satisfies $\lambda \leq \varpi_{\gamma}$ then $\lambda=\varpi_{\gamma}$.

Lemma. Let $\lambda \in P^{+} \backslash\{0\}$ and let $\gamma \in \Gamma$ be such that $\lambda=\varpi_{\gamma}(\bmod Q)$. Then $\lambda \geq \varpi_{\gamma}$. In addition, if $\lambda \in P^{+} \cap Q^{+}$and $\lambda \neq 0$, then $\lambda \geq \beta$ where $\beta=\theta$ if $\mathfrak{g}$ is simply laced and $\beta=\theta_{\text {s }}$ otherwise.

Proof. For $\mathfrak{g} \cong \mathfrak{s l}_{\ell+1}$ one can show by a direct computation that $\varpi_{i}+\varpi_{j} \in$ $\varpi_{i+j}(\bmod \ell+1)+Q^{+}, 1 \leq i, j \leq \ell$. The statement follows since the minimal representatives of the elements of $\Gamma$ in this case are $\varpi_{i}, 0 \leq i \leq \ell$, and any $\lambda \in P^{+} \backslash\{0\}$ can be written as $\lambda=\sum_{r=1}^{N} \varpi_{i_{r}}$ for some $N \geq 1$ and $1 \leq i_{r} \leq \ell$. For all other types the statement is trivially checked.

2.2. For $\lambda \in P^{+}$, let $V(\lambda)$ be the irreducible finite-dimensional $\mathfrak{g}$-module with highest weight vector $v_{\lambda}$, i.e. the cyclic module generated by $v_{\lambda}$ with defining relations:

$$
\mathfrak{n}^{+} v_{\lambda}=0, \quad h v_{\lambda}=\lambda(h) v_{\lambda}, \quad\left(x_{\alpha_{i}}^{-}\right)^{\lambda\left(\alpha_{i}^{\vee}\right)+1} v_{\lambda}=0,
$$

for all $i \in I, h \in \mathfrak{h}$. Given any $\mathfrak{g}$-module $M$ and $\mu \in \mathfrak{h}^{*}$ set

$$
M_{\mu}=\{v \in M: h v=\mu(h) v, h \in \mathfrak{h}\} .
$$

If $M$ is finite-dimensional, then $M=\bigoplus_{\mu \in \mathfrak{h}^{*}} M_{\mu}$, and moreover

$$
M \cong \bigoplus_{\lambda \in P^{+}} V(\lambda)^{\oplus m_{\lambda}(M)}, \quad m_{\lambda}(M) \in \mathbf{Z}_{+}
$$

The following Lemma is standard (see [11] for instance).

Lemma. Let $\lambda, \mu \in P^{+}$be such that $\lambda \geq \mu$. Then $V(\lambda)_{\mu} \neq 0$.

2.3. Given a Lie algebra $\mathfrak{a}$, let $\mathbf{U}(\mathfrak{a})$ denote the universal enveloping algebra of $\mathfrak{a}$ and let $L(\mathfrak{a})=\mathfrak{a} \otimes \mathbf{C}\left[t, t^{-1}\right]$ be the loop algebra of $\mathfrak{a}$ with the Lie bracket given by

$$
[x \otimes f, y \otimes g]=[x, y]_{\mathfrak{a}} \otimes f g,
$$

for all $x, y \in \mathfrak{a}, f, g \in \mathbf{C}\left[t^{ \pm 1}\right]$. The Lie algebra $L(\mathfrak{a})$ and its universal enveloping algebra are $\mathbf{Z}$-graded by the powers of $t$. We shall identify $\mathfrak{a}$ with the subalgebra $\mathfrak{a} \otimes 1$ of $L(\mathfrak{a})$. Denote by $L^{e}(\mathfrak{a})=L(\mathfrak{a}) \oplus \mathbf{C} d$ the extended loop algebra of $\mathfrak{a}$, in which $\left[d, x \otimes t^{n}\right]=n x \otimes t^{n}$.

2.4. Let $\mathfrak{h}_{e}=\mathfrak{h} \oplus \mathbf{C} d$, which is an abelian Lie subalgebra of $L^{e}(\mathfrak{g})$. Define $\delta \in \mathfrak{h}_{e}^{*}$ by

$$
\delta(d)=1, \quad \delta(h)=0, \quad \forall h \in \mathfrak{h} .
$$

We regard elements of $\mathfrak{h}^{*}$ as elements of $\mathfrak{h}_{e}^{*}$ by setting $\lambda(d)=0$ for $\lambda \in \mathfrak{h}^{*}$. In particular, we identify $P$ and $P^{+}$with their respective images in $\mathfrak{h}_{e}^{*}$. Obviously, $\mathfrak{h}_{e}^{*}=\mathfrak{h}^{*} \oplus \mathbf{C} \delta$. Set $P_{e}=P \oplus \mathbf{Z} \delta$ and let $P_{e}^{+}=P^{+} \oplus \mathbf{Z} \delta$. Then $P_{e} \subset\left\{\lambda \in \mathfrak{h}_{e}^{*}\right.$ : $\left.\lambda\left(\alpha_{i}^{\vee}\right) \in \mathbf{Z} \forall i \in I\right\}$.

Let $\widehat{W}$ be the affine Weyl group associated with $W$. Its image in the group of automorphisms of $\mathfrak{h}_{e}^{*}$ identifies with the semidirect product of $W$ with an abelian group generated by the $t_{h}, h \in \mathbf{Z}\left(W \theta^{\vee}\right)$ where $t_{h}(\lambda)=\lambda-\lambda(h) \delta$ for all $\lambda \in \mathfrak{h}_{e}^{*}$. 
Given $\lambda \in P^{+}$, let $r_{\lambda}=\min _{h \in \mathbf{Z}\left(W \theta^{\vee}\right)}\{\lambda(h): \lambda(h)>0\} \in \mathbf{N}$. This number exists since $\mathbf{Z}\left(W \theta^{\vee}\right)$ is contained in the $\mathbf{Z}$-span of the $\alpha_{i}^{\vee}, i \in I$. Then for all $s \in \mathbf{Z}$, there exists $w \in \widehat{W}$ such that $w(\lambda+s \delta)=\lambda+\bar{s} \delta$, where $0 \leq \bar{s}<r_{\lambda}$.

2.5. Given $i \in I$, let $\Lambda_{i}^{ \pm}(u) \in \mathbf{U}(L(\mathfrak{h}))[[u]]$ be defined by

$$
\Lambda_{i}^{ \pm}(u)=\sum_{k \geq 0} \Lambda_{i, \pm k} u^{k}=\exp \left(-\sum_{k>0} \frac{\alpha_{i}^{\vee} \otimes t^{ \pm k}}{k} u^{k}\right) .
$$

It is easy to see (cf. [8]) that $\mathbf{U}(L(\mathfrak{h}))$ is the polynomial algebra on $\Lambda_{i, \pm k}, i \in I$, $k \in \mathbf{Z}, k \neq 0$.

\section{Elementary PRoperties of the CATEgory $\mathcal{I}^{\text {fin }}$}

3.1. Recall that an $L^{e}(\mathfrak{g})$-module $V$ is said to be integrable if

$$
V=\bigoplus_{\mu \in \mathfrak{h}_{e}^{*}} V_{\mu}
$$

and the elements $x_{\alpha}^{ \pm} \otimes t^{s}$ act locally nilpotently on $V$ for all $\alpha \in R^{+}$and $s \in \mathbf{Z}$. Denote by $\mathcal{I}$ the category of integrable $L^{e}(\mathfrak{g})$-modules. Let $\mathrm{wt}^{e}(V)=\left\{\mu \in \mathfrak{h}_{e}^{*}\right.$ : $\left.V_{\mu} \neq 0\right\}$ be the set of weights of $V$ with respect to $\mathfrak{h}_{e}$. It is well-known that the set $\mathrm{wt}^{e}(V)$ is $\widehat{W}$-invariant. The following Lemma follows immediately from Section 2.4 and will be used repeatedly in the rest of the paper.

Lemma. Let $V \in \mathrm{Ob} \mathcal{I}$ and assume that $\mu+s \delta \in \mathrm{wt}^{e}(V)$ where $\mu \in P^{+}, s \in \mathbf{Z}$. Then $\mu+r \delta \in \mathrm{wt}^{e}(V)$ for some $0 \leq r<r_{\lambda}$.

3.2. For $a \in \mathbf{C}$ set

$$
V\{a\}=\{v \in V: d v=(a+k) v \text { for some } k \in \mathbf{Z}\} .
$$

It is trivial to check that $V\{a\}$ are $L^{e}(\mathfrak{g})$-submodules of $V$ and that $V\{a\}=V\{b\}$ if and only if $a-b \in \mathbf{Z}$. For any $\bar{a} \in \mathbf{C} / \mathbf{Z}$ set $V\{\bar{a}\}=V\{a\}$ where $a$ is any representative of $\bar{a}$. Let $\mathcal{I}\{\bar{a}\}, \bar{a} \in \mathbf{C} / \mathbf{Z}$, be the full subcategory of $\mathcal{I}$ whose objects are $L^{e}(\mathfrak{g})$-modules $V$ satisfying $V=V\{\bar{a}\}$.

Lemma. Let $V$ be an integrable $L^{e}(\mathfrak{g})$-module.

(i) Let $\mu \in \mathrm{wt}^{e}(V)$. Then $\mu\left(\alpha_{i}^{\vee}\right) \in \mathbf{Z}, i \in I$.

(ii) We have

$$
V=\bigoplus_{\bar{a} \in \mathbf{C} / \mathbf{Z}} V\{\bar{a}\}
$$

Moreover, if $V=V\{\bar{a}\}, V^{\prime}=V^{\prime}\{\bar{b}\}$ with $\bar{a} \neq \bar{b}$, then $\operatorname{Ext}_{\mathcal{I}}^{1}\left(V, V^{\prime}\right)=0$. In particular,

$$
\mathcal{I}=\bigoplus_{\bar{a} \in \mathbf{C} / \mathbf{Z}} \mathcal{I}\{\bar{a}\}
$$

and the categories $\mathcal{I}\{\bar{a}\}$ are equivalent for all $a \in \mathbf{C} / \mathbf{Z}$.

Proof. Part (i) is standard and follows from the representation theory of $\mathfrak{s l}_{2}$ applied to the subalgebras of $L^{e}(\mathfrak{g})$ spanned by the elements $\left\{x_{\alpha_{i}}^{ \pm}, \alpha_{i}^{\vee}\right\}$ for $i \in I$. The first two statements in (ii) are straightforward while for the last it is sufficient to observe that the functor $V \mapsto V \otimes \mathbf{C}_{-a \delta}$, where $\mathbf{C}_{-a \delta}$ is the 1-dimensional $L^{e}(\mathfrak{g})$-module on which $L(\mathfrak{g})$ acts trivially and $d$ acts by $-a$, provides an equivalence of categories between $\mathcal{I}\{\bar{a}\}$ and $\mathcal{I}\{\overline{0}\}$. 
It follows, in particular, that we can restrict ourselves to the subcategory $\mathcal{I}\{\overline{0}\}$ of $\mathcal{I}$. Observe that $V \in \mathrm{Ob} \mathcal{I}$ is an object in $\mathcal{I}\{\overline{0}\}$ if and only if $\mathrm{wt}^{e}(V) \subset P_{e}$. Let $\mathcal{I}^{\text {fin }}$ be the subcategory of $\mathcal{I}$ consisting of modules $V$ such that wt ${ }^{e}(V) \subset P_{e}$ and $\operatorname{dim} V_{\mu}<\infty$ for all $\mu \in P_{e}$.

3.3. Let $V$ be an integrable $L^{e}(\mathfrak{g})$-module. For $\lambda \in P_{e}^{+} \backslash \mathbf{Z} \delta$, set $V_{\lambda}^{+}=\left\{v \in V_{\lambda}\right.$ : $\left.L\left(\mathfrak{n}^{+}\right) v=0\right\}$ and

$$
V^{+}=\bigoplus_{\lambda \in P_{e}^{+} \backslash \mathbf{Z} \delta: 0 \leq \lambda(d)<r_{\lambda}} V_{\lambda}^{+}
$$

Proposition. Let $V$ be an object in $\mathcal{I}^{\text {fin }}$ and suppose that $V_{1} \subsetneq V_{2} \subsetneq \cdots$ is an ascending chain of $L^{e}(\mathfrak{g})$-submodules of $V$. Then $\mathrm{wt}^{e}\left(V_{r} / V_{r-1}\right) \subset \mathbf{Z} \delta$ for all but finitely many $r \geq 1$. In particular, $V^{+}$is finite-dimensional.

Proof. Write

$$
V=\bigoplus_{\gamma \in \Gamma} V[\gamma]
$$

where

$$
V[\gamma]=\bigoplus_{\mu=\varpi_{\gamma}} V_{\mu+n \delta} V
$$

Since $V[\gamma]$ is obviously an $L^{e}(\mathfrak{g})$-submodule of $V$ and $\Gamma$ is a finite group we can assume without loss of generality that $V=V[\gamma]$. Suppose for a contradiction that $\mathrm{wt}^{e}\left(V_{r} / V_{r-1}\right) \not \subset \mathbf{Z} \delta$ for infinitely many $r \geq 1$. Since $V$ is integrable, $V_{r} / V_{r-1}$ is a (possibly infinite) direct sum of finite-dimensional $\mathfrak{g}$-modules. In particular, for infinitely many $r \geq 1$ there exists $\mu_{r} \in P^{+} \backslash\{0\}$ and $s_{r} \in \mathbf{Z}$ such that $v \in$ $\left(V_{r} / V_{r-1}\right)_{\mu_{r}+s_{r} \delta}, v \neq 0$ generates a simple highest weight $\mathfrak{g}$-submodule isomorphic to $V\left(\mu_{r}\right)$. By Lemma 2.1, $\mu_{r} \geq \varpi_{\gamma}$, hence $V\left(\mu_{r}\right)_{\varpi_{\gamma}} \neq 0$ by Lemma 2.2 , and we conclude that $\varpi_{\gamma}+s_{r} \delta \in \mathrm{wt}^{e}\left(V_{r} / V_{r-1}\right)$.

Suppose first that $\varpi_{\gamma} \neq 0$. Then by Lemma 3.1, $\varpi_{\gamma}+\bar{s}_{r} \delta \in \mathrm{wt}^{e}\left(V_{r} / V_{r-1}\right)$ with $0 \leq \bar{s}_{r}<r_{\varpi_{\gamma}}$. It follows that there exists $0 \leq s<r_{\varpi_{\gamma}}$ such that $\varpi_{\gamma}+s \delta \in$ $\mathrm{wt}^{e}\left(V_{r} / V_{r-1}\right)$ for infinitely many $r \geq 1$. This implies that $V_{\varpi_{\gamma}+s \delta}$ is infinite dimensional, which is clearly a contradiction. If $\varpi_{\gamma}=0$ and $\mu_{r} \neq 0$, then by Lemma 2.1 we have that $\mu_{r} \geq \beta$ where $\beta=\theta$ or $\beta=\theta_{s}$. The preceding argument would then imply that $V_{\beta+s \delta}$ is infinite dimensional for some $0 \leq s<r_{\beta}$, which is again a contradiction.

3.4. In the category $\mathcal{I}^{\text {fin }}$ the role similar to that of highest weight modules is played by $\ell$-highest weight modules.

Definition. Let $V$ be an integrable module. A non-zero element $v \in V_{\lambda}^{+}$is called an $\ell$-highest weight vector if $\mathbf{U}\left(L^{e}(\mathfrak{h})\right) v$ is an indecomposable $\mathbf{U}\left(L^{e}(\mathfrak{h})\right)$-submodule of $V$ and if $\operatorname{dim}\left(\mathbf{U}\left(L^{e}(\mathfrak{h})\right) v\right)_{\lambda+r \delta} \leq 1$ for all $r \in \mathbf{Z}$. We say that $V$ is $\ell$-highest weight if $V=\mathbf{U}\left(L^{e}(\mathfrak{g})\right) v$ where $v \in V$ is an $\ell$-highest weight vector.

It is not hard to see by the usual arguments that an $\ell$-highest weight module is indecomposable. Observe also that if $v \in V_{\lambda}^{+}$and $\mathbf{U}\left(L^{e}(\mathfrak{h})\right) v$ is a simple $\mathbf{U}\left(L^{e}(\mathfrak{h})\right)$ module, then $\operatorname{dim}\left(\mathbf{U}\left(L^{e}(\mathfrak{h})\right) v\right)_{\lambda+r \delta} \leq 1$ and so $v$ is an $\ell$-highest weight vector. 
3.5. Retain the assumption of Section 3.4 and consider $V^{L(\mathfrak{g})}=\{v \in V: L(\mathfrak{g}) v=$ $0\}$. Obviously, $V^{L(\mathfrak{g})}$ is an $L^{e}(\mathfrak{g})$-submodule of $V$.

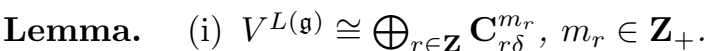

(ii) Suppose that $\mathrm{wt}^{e}(V) \subset \mathbf{Z} \delta$. Then $V=V^{L(\mathfrak{g})}$.

(iii) Suppose that $V \neq V^{L(\mathfrak{g})}$. Then $V / V^{L(\mathfrak{g})}$ does not admit an $L^{e}(\mathfrak{g})$-submodule isomorphic to $\mathbf{C}_{r \delta}, r \in \mathbf{Z}$.

Proof. Parts (i) and (ii) are immediate. For (iii), let $V_{0}=V^{L(\mathfrak{g})} \subsetneq V$ and suppose that there exists $v \in V \backslash V_{0}$, such that $L(\mathfrak{g}) v \subset V_{0}$. Since $V$ is a weight module, we can write, uniquely, $v=\sum_{k} v_{\mu_{k}}, \mu_{k} \in \mathfrak{h}_{e}^{*}$. Since $h v \in V_{0}$ for all $h \in \mathfrak{h}_{e}$ and $\mathrm{wt}^{e}\left(V_{0}\right) \subset \mathbf{Z} \delta$ by (i), it follows that $\mu_{k} \in \mathbf{Z} \delta$. Then $\left(x_{\alpha}^{ \pm} \otimes t^{n}\right) v=0$ and so $L(\mathfrak{g}) v=0$. Thus, $v \in V_{0}$, which is a contradiction.

\section{Finiteness in the CAtegory $\mathcal{I}^{\text {fin }}$}

The main result of this section is the following theorem. Although we state and prove this result only for the classical affine Lie algebras, it is clear that the proof goes over verbatim to the quantum case.

Theorem 1. Let $V$ be an object in $\mathcal{I}^{\text {fin }}$. Then $V$ is isomorphic to a direct sum of indecomposable modules. More precisely, there exists submodules $U_{j}, j=1,2$, such that $V=U_{1} \oplus U_{2}$ with $U_{1} \subset V^{L(\mathfrak{g})}, U_{2}^{+}=V^{+}$. Moreover, $U_{2}$ is isomorphic to a finite direct sum of indecomposable modules.

We prove this result in the rest of the section.

Remark. Note that there exist indecomposable modules of infinite length in $\mathcal{I}^{\text {fin }}$. For example, let $M=\mathfrak{g} \oplus \mathbf{C}$ as a $\mathfrak{g}$-module with the $L(\mathfrak{g})$-module structure defined by $\left(x \otimes t^{k}\right)(y \oplus a)=[x, y] \oplus k(x, y)_{\mathfrak{g}}$, where $(\cdot, \cdot)_{\mathfrak{g}}$ is the Killing form of $\mathfrak{g}$, for all $x, y \in \mathfrak{g}, k \in \mathbf{Z}$ and $a \in \mathbf{C}$. Then $M$ is an indecomposable $L(\mathfrak{g})$-module. Applying the functor $L$ (cf. Section 5.4) we conclude that $L(M)$ contains $L(\mathbf{C})=\bigoplus_{r \in \mathbf{Z}} \mathbf{C}_{r \delta}$ as a submodule and $L(M) / L(\mathbf{C}) \cong L(\mathfrak{g})$ which is a simple $L^{e}(\mathfrak{g})$-module. Thus, $L(M)$ has infinite length and it is easy to see that $L(M)$ is indecomposable.

4.1. The following proposition was established in [3] (Proposition 3.2) in the quantum case. We provide its proof here for the sake of completeness.

Proposition. Let $V$ be an object in $\mathcal{I}^{\text {fin }}$. Then there exists $\lambda \in \mathrm{wt}^{e}(V)$ such that $\lambda+\eta \notin \mathrm{wt}^{e}(V)$ for all $\eta \in Q^{+} \backslash\{0\}$. In particular, $\lambda \in P_{e}^{+}$and $V_{\lambda}^{+} \neq 0$.

Proof. By Lemma 3.5, we may assume that $\mathrm{wt}^{e}(V) \not \subset \mathbf{Z} \delta$. Suppose that for each $\mu \in$ $\mathrm{wt}^{e}(V)$ there exists $\nu \in Q^{+}$such that $\mu+\nu \in \mathrm{wt}^{e}(V)$. Fix some $\mu \in \mathrm{wt}^{e}(V)$. Then there exists an infinite sequence $\left\{\eta_{r}\right\}_{r \geq 1}$ such that $\eta_{r} \leq \eta_{r+1}$ and $\mu+\eta_{r} \in \mathrm{wt}^{e}(V)$ for all $r \geq 1$. Set $W_{r}:=\mathbf{U}(\mathfrak{g}) V_{\mu+\eta_{r}}$. Then $W_{r}$ is an integrable $\mathfrak{g}$-module with finitedimensional weight spaces and hence is isomorphic to a finite direct sum of simple finite-dimensional $\mathfrak{g}$-modules $V\left(\mu_{r, s}\right)$ for some $\mu_{r, s} \in P^{+}$. Choose $s_{1}$ such that $\nu_{1}:=\mu_{1, s_{1}}>\mu$. Such $s_{1}$ exists since $\mu+\eta_{1}$ is a weight of $W_{1}$. Furthermore, let $r_{2}$ be the smallest positive integer so that there exists $s_{2}$ with $\nu_{2}:=\mu_{r_{2}, s_{2}}>\mu, \mu_{1, s_{1}} \neq$ $\mu_{r_{2}, s_{2}}$. Notice that $r_{2}$ always exists since the module $W_{1}$ is finite-dimensional and the maximal weights which occur in $W_{r}$ keep increasing. Repeating this process, we obtain an infinite collection of elements $\nu_{k}>\mu, k \geq 1$, such that $V\left(\nu_{k}\right)$ is isomorphic to an irreducible $\mathfrak{g}$-submodule $W\left(\nu_{k}\right)$ of $V$. By Lemma 2.2 it follows that 
$V_{\mu} \cap W\left(\nu_{k}\right) \neq 0$ for all $k \geq 1$. Since all the $\nu_{k}$ are distinct, the sum of $W\left(\nu_{k}\right)$ is direct, which contradicts the finite-dimensionality of $V_{\mu}$. In particular $\lambda+\alpha_{i} \notin \mathrm{wt}^{e}(V)$ for all $i \in I$ which implies that $\lambda \in P_{e}^{+}$.

4.2 .

Proposition. Suppose that $V$ is an object in $\mathcal{I}^{\text {fin }}$ and assume that $\mathrm{wt}^{e}(V)$ is not a subset of $\mathbf{Z} \delta$. Then $V$ contains an $\ell$-highest weight submodule generated by $v \in V_{\lambda+r \delta}^{+}$for some $\lambda \in P^{+} \backslash\{0\}$ and $0 \leq r<r_{\lambda}$.

Proof. By Proposition 4.1 and Lemma 3.1 we find that there exists $\lambda \in P^{+} \backslash\{0\}$ and $0 \leq s<r_{\lambda}$ such that $V_{\lambda+s \delta}^{+} \neq 0$. Fix a non-zero $v \in V_{\lambda+s \delta}^{+}$. Let $V_{1}=\mathbf{U}\left(L^{e}(\mathfrak{g})\right) v$. We claim that $V_{1}$ and hence $V$ contains an $\ell$-highest weight $L^{e}(\mathfrak{g})$-submodule. For this it suffices to prove that the $\mathbf{U}\left(L^{e}(\mathfrak{h})\right)$-module $\bigoplus_{r \in \mathbf{Z}}\left(V_{1}\right)_{\lambda+r \delta}$ contains an irreducible $\mathbf{U}\left(L^{e}(\mathfrak{h})\right)$-submodule. If not, then there exist integers $r_{k} \in \mathbf{Z}$, and non-zero elements $v_{k} \in\left(V_{1}\right)_{\lambda+r_{k} \delta}, k \in \mathbf{N}$, such that $\mathbf{U}\left(L^{e}(\mathfrak{h})\right) v_{k} \supsetneq \mathbf{U}\left(L^{e}(\mathfrak{h})\right) v_{k+1}$ and we can assume as usual that $0 \leq r_{k}<r_{\lambda}$ for all $k$. Since the $v_{k}$ are obviously linearly independent, this contradicts the fact that the weight spaces $V_{\lambda+s \delta}$ are finite dimensional. Thus, there exists an element $v_{0} \in V_{\mu}^{+} \backslash\{0\}$ for some $\mu \in \mathrm{wt}^{e}(V)$ such that $\mathbf{U}\left(L^{e}(\mathfrak{h})\right) v_{0}$ is a simple $\mathbf{U}\left(L^{e}(\mathfrak{h})\right)$-module. Then $\mathbf{U}\left(L^{e}(\mathfrak{g})\right) v_{0}$ is an $\ell$-highest weight module.

Corollary. Suppose that $V \in \mathrm{Ob} \mathcal{I}^{\text {fin }}$. Then $V$ contains a simple $\ell$-highest weight submodule.

Proof. By Lemma 3.5, we may assume that $\mathrm{wt}^{e}(V)$ is not a subset of $\mathbf{Z} \delta$. By the above Proposition, it is enough to consider the case when $V$ is an $\ell$-highest weight module generated by $v \in V_{\lambda+k \delta}^{+}, \lambda \in P^{+}, 0 \leq k<r_{\lambda}$. Assume for a contradiction that the Corollary is false. Then there exists an infinite family of non-zero $\ell$-highest weight $L^{e}(\mathfrak{g})$-submodules $V_{r}, r>0$ of $V$, such that $V_{r} \supsetneq V_{r+1}$. Let $v_{r}$ be an $\ell$-highest weight vector generating $V_{r}$ and assume that the weight of $v_{r}$ is $\mu_{r}+s_{r} \delta$, where $\mu_{r} \in P^{+} \cap\left(\lambda-Q^{+}\right), s_{r} \in \mathbf{Z}$. As before, we may also assume that $0 \leq s_{r}<r_{\mu_{r}}$. Since the set $P^{+} \cap\left(\lambda-Q^{+}\right)$is finite, the weights of the linearly independent elements $v_{r}, r \geq 1$, are contained in a finite set, which is a contradiction since weight spaces of $V$ are finite dimensional.

4.3. The next Proposition provides an analogue of the finite length property for the category $\mathcal{I}^{\text {fin }}$.

Definition. We say that a descending chain of $L^{e}(\mathfrak{g})$-submodules $V=V_{N} \supsetneq$ $V_{N-1} \supsetneq \cdots \supsetneq V_{1} \supsetneq V_{0}=0$ of an $L^{e}(\mathfrak{g})$-module $V \in \mathrm{ObI}^{\text {fin }}$ is a pseudo-JordanHölder series, if

(i) either $V_{k} / V_{k-1}$ is a simple $\ell$-highest weight module and $\mathrm{wt}^{e}\left(V_{k} / V_{k-1}\right) \not \subset \mathbf{Z} \delta$,

(ii) or $V_{k} / V_{k-1} \cong \bigoplus_{r \in \mathbf{Z}} \mathbf{C}_{r \delta}^{m_{r}}$ for some $m_{r} \in \mathbf{Z}_{+}$

and for all $k$, at most one of $V_{k} / V_{k-1}, V_{k+1} / V_{k}$ satisfies (ii).

Proposition. Let $V \in \mathrm{Ob} \mathcal{I}^{\text {fin }}$ be indecomposable. Then $V$ admits a pseudoJordan-Hölder series. In particular, $V$ has finite length, in the usual sense, if and only if the set of $r \in \mathbf{Z}$ such that $\mathbf{C}_{r \delta}$ is a subquotient of $V$ is finite.

Proof. Since $V$ is indecomposable $\mathrm{wt}^{e}(V) \not \subset \mathbf{Z} \delta$. If $V^{L(\mathfrak{g})} \neq 0$, set $V_{1}=V^{L(\mathfrak{g})}$. Otherwise, by Corollary 4.2, choose $V_{1}$ to be a simple $\ell$-highest weight submodule generated by an element of $V_{\lambda_{1}+r \delta}^{+}$for some $\lambda_{1} \in P^{+} \backslash\{0\}$ and $0 \leq r<r_{\lambda}$. 
This procedure can obviously be repeated to get an ascending chain satisfying the conditions of the above definition. Proposition 3.3 implies that we must reach a stage when $\left(V / V_{N}\right)^{L(\mathfrak{g})}=V / V_{N}$ and hence $V_{N+1}=V$, which proves the finiteness condition of the proposition.

4.4. Let $V$ be an object in $\mathcal{I}^{\text {fin }}$ and let $V=V_{N} \supseteq \cdots \supseteq V_{1} \supseteq V_{0}=0$ and $V=V_{N^{\prime}}^{\prime} \supseteq \cdots \supseteq V_{1}^{\prime} \supseteq V_{0}^{\prime}=0$ be descending chains of its $L^{e}(\mathfrak{g})$-submodules. We call these chains equivalent if

(i) $\left|\left\{0<i \leq N: \mathrm{wt}^{e}\left(V_{i} / V_{i-1}\right) \not \subset \mathbf{Z} \delta\right\}\right|=\left|\left\{0<j \leq N^{\prime}: \mathrm{wt}^{e}\left(V_{j}^{\prime} / V_{j-1}^{\prime}\right) \not \subset \mathbf{Z} \delta\right\}\right|$.

(ii) For each $0<i \leq N$ such that $\mathrm{wt}^{e}\left(V_{i} / V_{i-1}\right) \not \subset \mathbf{Z} \delta$, there exists $0<j \leq N^{\prime}$ such that $V_{i} / V_{i-1} \cong V_{j}^{\prime} / V_{j-1}^{\prime}$.

It is easy to see that the above relation is indeed an equivalence.

Proposition. Let $V$ be an indecomposable object in $\mathcal{I}^{\text {fin }}$. Then its pseudo-JordanHölder series is unique up to equivalence defined above.

Proof. It is easy to see that any refinement of a pseudo-Jordan-Hölder series for an indecomposable object $V$ in $\mathcal{I}^{\text {fin }}$ is equivalent, in the above sense, to that pseudoJordan-Hölder series itself. The statement follows immediately from the Schreier Refinement Theorem.

It follows from the above proposition that the number

$$
\left|\left\{0<i \leq N: \mathrm{wt}^{e}\left(V_{i} / V_{i-1}\right) \not \subset \mathbf{Z} \delta\right\}\right|,
$$

where $V=V_{N} \supsetneq \cdots \supsetneq V_{1} \supsetneq V_{0}=0$ is a pseudo-Jordan-Hölder series for $V$, is well-defined. We call this number the pseudo-length of $V$.

\subsection{We can now prove Theorem 1 .}

Proof. Let $V \in \mathrm{Ob} \mathcal{I}^{\text {fin }}$. For each $s \in \mathbf{Z}$, let $m_{s} \in \mathbf{Z}_{+}$be the multiplicity of $\mathbf{C}_{s \delta}$ as a direct summand of $V$ and set $U_{1}=\bigoplus_{r \in \mathbf{Z}} \mathbf{C}_{r \delta}^{m_{r}}$. Clearly $U_{1} \subset V^{L(\mathfrak{g})}$. For $s \in \mathbf{Z}$ with $m_{s} \neq 0$ fix an $L^{e}(\mathfrak{g})$-module complement $V^{(s)}$ of $\mathbf{C}_{s \delta}^{m_{s}}$ in $V$ and let $U_{2}=\bigcap_{r \in \mathbf{Z}: m_{r} \neq 0} V^{(r)}$. Since $V^{(r)} \cap \mathbf{C}_{r \delta}^{m_{r}}=0$ it follows that $U_{1} \cap U_{2}=\{0\}$.

Let $v \in V_{\mu}, \mu \in \mathrm{wt}^{e}(V)$. If $\mu \neq s \delta$ for some $s \in \mathbf{Z}$ such that $m_{s} \neq 0$, then $v \in V^{(s)}$ for all $s \in \mathbf{Z}$ and so $v \in U_{2}$. Otherwise, since $V=V^{(s)} \oplus \mathbf{C}_{s \delta}^{m_{s}}$, we can write $v$ uniquely as $v=u_{1}+u_{2}$ for some $u_{1} \in \mathbf{C}_{s \delta}^{m_{s}}$ and $u_{2} \in V^{(s)}$. It follows from weight considerations that $u_{2} \in U_{2}$ and so we have proved that $V=U_{1} \oplus U_{2}$. Note that if $U_{2} \neq 0$, then by Lemma $3.5 \mathrm{wt}^{e}\left(U_{2}\right)$ is not a subset of $\mathbf{Z} \delta$.

It remains to show that $U_{2}$ is a finite direct sum of indecomposable $L^{e}(\mathfrak{g})$ modules. If $U_{2}$ is indecomposable we are done. Otherwise, we can write $U_{2}=$ $M_{1} \oplus M_{2}$. Note that $\mathrm{wt}^{e}\left(M_{j}\right)$ is not a subset of $\mathbf{Z} \delta$ for $j=1,2$ since otherwise by Lemma 3.5 we would have a contradiction to the definition of $U_{1}$. Hence $\operatorname{dim} M_{j}^{+}>$ 0 for $j=1,2$, and since $U_{2}^{+}=M_{1}^{+} \oplus M_{2}^{+}$, $\operatorname{dim} U_{2}^{+}=\operatorname{dim} M_{1}^{+}+\operatorname{dim} M_{2}^{+}$. The statement follows by repeating the argument and using the fact that $\operatorname{dim} U_{2}^{+}<\infty$ (cf. Proposition 3.3).

Since by Theorem 1 every object in the category $\mathcal{I}^{\text {fin }}$ is a direct sum of indecomposables, it makes sense to define the blocks in that category. 
4.6. Let $\mathcal{C}$ be an abelian category in which any object is a direct sum of indecomposables. We say that two indecomposable objects $U_{i}, i=1,2$, in a $\mathcal{C}$ are linked and write $U_{1} \sim U_{2}$ if there do not exist full abelian subcategories $\mathcal{C}_{i}, i=1,2$, such that $U_{i} \in \mathrm{Ob}_{i}$ and $\mathcal{C}=\mathcal{C}_{1} \oplus \mathcal{C}_{2}$. If the $U_{i}$ are decomposable, then they are said to be linked if every indecomposable summand of $U_{1}$ is linked to every indecomposable summand of $U_{2}$. This defines an equivalence relation on $\mathcal{C}$, and the equivalence classes are called blocks. Each block is a full abelian subcategory, and the category $\mathcal{C}$ is a direct sum of the blocks.

It is not hard to see that the following is an equivalent definition of linking. Two indecomposable objects $U, V$ in $\mathcal{C}$ are linked if and only if there exists a family of indecomposable objects $U_{1}=U, U_{2}, \ldots, U_{l}=V$ in $\mathcal{C}$ such that either $\operatorname{Hom}_{\mathcal{C}}\left(U_{k}, U_{k+1}\right) \neq 0$ or $\operatorname{Hom}_{\mathcal{C}}\left(U_{k+1}, U_{k}\right) \neq 0$ for all $k=1, \ldots, l-1$.

4.7. Following [5], let $\Xi$ be the set of functions $\chi: \mathbf{C}^{\times} \rightarrow \Gamma$ with finite support. The addition of functions defines on $\Xi$ the structure of an abelian group. Given $i \in I, a \in \mathbf{C}^{\times}$, set $\chi_{i, a}(z)=\delta_{a, z} \bar{\varpi}_{i}$, where $\bar{\varpi}_{i}$ denotes the canonical image of $\varpi_{i}$ in $\Gamma$. Clearly $\Xi$ is the free abelian group generated by the $\chi_{i, a}, i \in I, a \in \mathbf{C}^{\times}$.

Define an action of $\mathbf{C}^{\times}$on $\Xi$ by

$$
(a \cdot \chi)(z):=\chi(a z), \quad a, z \in \mathbf{C}^{\times} .
$$

Let $\bar{\Xi}$ be the set of orbits in $\Xi$ for this action and let $\bar{\chi}$ be the $\mathbf{C}^{\times}$-orbit of $\chi \in \Xi$. In the rest of the paper, we shall prove the following:

Theorem 2. The blocks in the category $\mathcal{I}^{\text {fin }}$ are parametrized by the elements of $\Xi$.

\section{The CATEGory $\mathcal{F}$ AND THE FUnCtOR $L$}

5.1. Let $\mathcal{P}^{+}$be the set of $I$-tuples of polynomials $\boldsymbol{\pi}=\left(\pi_{i}\right)_{i \in I}$ in $u$ with constant term 1 . We regard $\mathcal{P}^{+}$as a commutative monoid with multiplication defined component-wise. Let $\mathbf{1}=(1, \ldots, 1)$ and, for $i \in I$ and $a \in \mathbf{C}^{\times}$, let $\varpi_{i, a} \in \mathcal{P}^{+}$ be the $I$-tuple of polynomials with the polynomial $(1-a u)$ in the $i^{\text {th }}$ place and one everywhere else. The elements $\varpi_{i, a}, i \in I$, and $a \in \mathbf{C}^{\times}$generate the monoid $\mathcal{P}^{+}$. For $\boldsymbol{\pi} \in \mathcal{P}^{+}$, set $\lambda_{\boldsymbol{\pi}}=\sum_{i \in I}\left(\operatorname{deg} \pi_{i}\right) \varpi_{i} \in P^{+}$. Conversely, given $\lambda \in P^{+}$and $a \in \mathbf{C}^{\times}$, set $\boldsymbol{\pi}_{\lambda, a}=\prod_{i \in I} \varpi_{i, a}^{\lambda\left(\alpha_{i}^{\vee}\right)}$.

We say that $\boldsymbol{\pi}=\left(\pi_{i}\right)_{i \in I}, \boldsymbol{\pi}^{\prime}=\left(\pi_{i}^{\prime}\right)_{i \in I} \in \mathcal{P}^{+}$are co-prime if for all $i, j \in I$, the polynomials $\pi_{i}$ and $\pi_{j}^{\prime}$ are co-prime. Clearly any $\boldsymbol{\pi} \in \mathcal{P}^{+}$can be written, uniquely, as a product $\boldsymbol{\pi}_{\lambda_{j}, a_{j}}$ for some $\lambda_{j} \in P^{+}$and $a_{j} \in \mathbf{C}^{\times}, 1 \leq j \leq k$, and $a_{r} \neq a_{j}$ if $r \neq j$.

Let $m: \mathcal{P}^{+} \rightarrow \mathbf{Z}_{+}$be the map defined by setting $m(\boldsymbol{\pi})$ to be the maximal non-negative integer $r$ such that $\pi_{i} \in \mathbf{C}\left[u^{r}\right]$ for all $i \in I$.

Lemma. Suppose that $\lambda, \mu \in P^{+}$and $\lambda>\mu$. Let $\boldsymbol{\pi} \in \mathcal{P}^{+}$and $a \in \mathbf{C}^{\times}$be such that $\varpi_{i, a}$ is coprime to $\boldsymbol{\pi}$ for some $i \in I$. Then either $m\left(\boldsymbol{\pi} \boldsymbol{\pi}_{\lambda, a}\right)=1$ or $m\left(\boldsymbol{\pi} \boldsymbol{\pi}_{\mu, a}\right)=1$.

Proof. Observe that for all $\boldsymbol{\pi}^{\prime}=\left(\pi_{i}^{\prime}\right)_{i \in I} \in \mathcal{P}^{+}, m\left(\boldsymbol{\pi}^{\prime}\right)>1$ implies that $\sum_{r} \mu_{r}(h) b_{r}$ $=0$ for all $h \in \mathfrak{h}$, provided that $\boldsymbol{\pi}^{\prime}=\prod_{r} \boldsymbol{\pi}_{\mu_{r}, b_{r}}$ where the $b_{r}$ are distinct and $\mu_{r} \in$ $P^{+}$. Indeed, for $i \in I, \sum_{r} \mu_{r}\left(\alpha_{i}^{\vee}\right) b_{r}$ is obviously the coefficient of $u$ in the polynomial $\pi_{i}^{\prime}(u)$. Since we assume that $m\left(\boldsymbol{\pi}^{\prime}\right)>1$, it follows from the definition of $m\left(\boldsymbol{\pi}^{\prime}\right)$ that the coefficient of $u$ in all the $\pi_{i}^{\prime}, i \in I$, must be zero. 
Write $\boldsymbol{\pi}=\prod_{r=1}^{k} \boldsymbol{\pi}_{\lambda_{r}, a_{r}}$ where the $a_{r}$ are distinct. Obviously, $a \neq a_{r}$ for all $1 \leq$ $r \leq k$. Let $\beta=\lambda-\mu \in Q^{+} \backslash\{0\}$. Suppose that $m\left(\boldsymbol{\pi}_{\lambda} \boldsymbol{\pi}\right)>1$. By the above argument, $\lambda(h) a+\sum_{r} \lambda_{r}(h) a_{r}=0$ for all $h \in \mathfrak{h}$. Since $\beta \neq 0$, there exists $i \in I$ such that $\beta\left(\alpha_{i}^{\vee}\right) \neq 0$. Then $\mu\left(\alpha_{i}^{\vee}\right) a+\sum_{r} \lambda_{r}\left(\alpha_{i}^{\vee}\right) a_{r}=(\lambda-\beta)\left(\alpha_{i}^{\vee}\right) a+\sum_{r} \lambda_{r}\left(\alpha_{i}^{\vee}\right) a_{r}=$ $-\beta\left(\alpha_{i}^{\vee}\right) a \neq 0$ which implies $m\left(\boldsymbol{\pi}_{\mu} \boldsymbol{\pi}\right)=1$.

5.2 .

Definition. Let $V$ be an $L(\mathfrak{g})$-module and let $0 \neq v \in V$. We say that $v$ is an $\ell$-highest vector if

$$
L\left(\mathfrak{n}^{+}\right) v=0, \quad \operatorname{dim}_{\mathbf{C}} \mathbf{U}(L(\mathfrak{h})) v=1 .
$$

The module $V$ is said to be $\ell$-highest weight if it is generated, as an $L(\mathfrak{g})$-module, by an $\ell$-highest weight vector.

Given $\boldsymbol{\pi}=\left(\pi_{i}\right)_{i \in I} \in P^{+}$, let $\mathcal{W}(\boldsymbol{\pi})$ be the $L(\mathfrak{g})$-module generated by a vector $w_{\boldsymbol{\pi}}$ satisfying the relations:

$$
L\left(\mathfrak{n}^{+}\right) w_{\boldsymbol{\pi}}=0, \quad h w_{\boldsymbol{\pi}}=\lambda_{\boldsymbol{\pi}}(h) w_{\boldsymbol{\pi}}, \quad \Lambda_{i, \pm k} w_{\boldsymbol{\pi}}=\pi_{i, k}^{ \pm} w_{\boldsymbol{\pi}},
$$

where $h \in \mathfrak{h}, \pi_{i}(u)=\sum_{k \geq 0} \pi_{i, k}^{+} u^{k}$ and

$$
\sum_{k \geq 0} \pi_{i, k}^{-} u^{k}=u^{\operatorname{deg} \pi_{i}} \pi_{i}\left(u^{-1}\right) /\left(\left.u^{\operatorname{deg} \pi_{i}} \pi_{i}\left(u^{-1}\right)\right|_{u=0}\right) .
$$

The modules $\mathcal{W}(\boldsymbol{\pi})$ are clearly $\ell$-highest weight modules for $L(\mathfrak{g})$.

Let $\mathcal{F}$ be the category of finite-dimensional representations of $L(\mathfrak{g})$. The following was proved in $[2,7,8]$.

Proposition. (i) For all $\boldsymbol{\pi} \in \mathcal{P}^{+}, \mathcal{W}(\boldsymbol{\pi})$ is an indecomposable object in $\mathcal{F}$.

(ii) Any $\ell$-highest weight module in $\mathcal{F}$ is a quotient of $\mathcal{W}(\boldsymbol{\pi})$ for some $\boldsymbol{\pi} \in \mathcal{P}^{+}$.

(iii) The modules $\mathcal{W}(\boldsymbol{\pi})$ have a unique irreducible quotient $\mathcal{V}(\boldsymbol{\pi})$ and any irreducible module in $\mathcal{F}$ is isomorphic to $\mathcal{V}(\boldsymbol{\pi})$ for some $\boldsymbol{\pi} \in \mathcal{P}^{+}$.

(iv) Let $\boldsymbol{\pi}, \boldsymbol{\pi}^{\prime} \in \mathcal{P}^{+}$be coprime. Then $\mathcal{W}(\boldsymbol{\pi}) \otimes \mathcal{W}(\boldsymbol{\pi}) \cong \mathcal{W}\left(\boldsymbol{\pi} \boldsymbol{\pi}^{\prime}\right)$. In particular, any quotient of $\mathcal{W}\left(\boldsymbol{\pi} \boldsymbol{\pi}^{\prime}\right)$ is isomorphic to a tensor product of quotients of $\mathcal{W}(\boldsymbol{\pi})$ and $\mathcal{W}\left(\boldsymbol{\pi}^{\prime}\right)$

(v) Let $a \in \mathbf{C}^{\times}$and let $\tau_{a}$ be the automorphism of $L(\mathfrak{g})$ defined by $\tau_{a}\left(x \otimes t^{r}\right)=$ $a^{r} x \otimes t^{r}$ for all $x \in \mathfrak{g}, r \in \mathbf{Z}$. Then $\tau_{a}^{*} \mathcal{W}(\boldsymbol{\pi})$ is isomorphic, as an $L(\mathfrak{g})$-module, to $\mathcal{W}(\boldsymbol{\pi}(a u))$.

Corollary. Let $\boldsymbol{\pi} \in \mathcal{P}^{+}$and suppose that $m=m(\boldsymbol{\pi})>1$. Then there exists $\eta_{m} \in$ $\operatorname{End}_{\mathbf{C}} \mathcal{W}(\boldsymbol{\pi})$ such that $\eta_{m}\left(w_{\boldsymbol{\pi}}\right)=w_{\boldsymbol{\pi}}$ and $\eta_{m}\left(\left(x \otimes t^{r}\right) w\right)=\zeta_{m}^{-r}\left(x \otimes t^{r}\right) \eta_{\boldsymbol{\pi}}(w)$ for all $x \in \mathfrak{g}, r \in \mathbf{Z}$ and $w \in \mathcal{W}(\boldsymbol{\pi})$, where $\zeta_{m}$ is an $m^{\text {th }}$ primitive complex root of unity. Moreover, $\eta_{m}$ is of order $m$.

Proof. Let $a \in \mathbf{C}^{\times}$. Since $\tau_{a}^{*} \mathcal{W}(\boldsymbol{\pi})$ is isomorphic to $\mathcal{W}(\boldsymbol{\pi})$ as a vector space, it follows from (v) that there exists a map $\eta_{\boldsymbol{\pi}, a} \in \operatorname{Hom}_{\mathbf{C}}(\mathcal{W}(\boldsymbol{\pi}), \mathcal{W}(\boldsymbol{\pi}(a u)))$ such that $\eta_{\boldsymbol{\pi}, a}\left(w_{\boldsymbol{\pi}}\right)=w_{\boldsymbol{\pi}}$ and $\eta_{\boldsymbol{\pi}, a}\left(\left(x \otimes t^{r}\right) w\right)=a^{r}\left(x \otimes t^{r}\right) \eta_{\boldsymbol{\pi}, a}(w)$ for all $x \in \mathfrak{g}, r \in \mathbf{Z}$ and $w \in W$. Set $\eta_{m}=\eta_{\boldsymbol{\pi}, \zeta_{m}^{-1}}$. Since $m=m(\boldsymbol{\pi})>1, \boldsymbol{\pi}\left(\zeta_{m} u\right)=\boldsymbol{\pi}(u)$ and so $\eta_{m} \in \operatorname{End}_{\mathbf{C}} \mathcal{W}(\boldsymbol{\pi})$. The first two properties of $\eta_{m}$ are immediate. For the last, observe that since $\mathcal{W}(\boldsymbol{\pi})$ is generated by $w_{\boldsymbol{\pi}}$ as an $L(\mathfrak{g})$-module, it follows from the properties of $\eta_{m}$ that $\mathcal{W}(\boldsymbol{\pi})$ is a direct sum of eigenspaces of $\eta_{m}$, all eigenvalues of $\eta_{m}$ are $m^{t h}$ complex roots of unity, and $\zeta_{m}$ is an eigenvalue of $\eta_{m}$. 
Remark. The module $\mathcal{V}\left(\boldsymbol{\pi}_{\lambda, a}\right)$ is isomorphic to $V(\lambda)$ as a $\mathfrak{g}$-module, the $L(\mathfrak{g})$-module structure being defined by the evaluation at $a$, that is, $\left(x \otimes t^{n}\right) v=a^{n} x v$ for all $x \in \mathfrak{g}$, $n \in \mathbf{Z}$ and $v \in V(\lambda)$. More generally, $\mathcal{V}(\boldsymbol{\pi}), \boldsymbol{\pi} \in \mathcal{P}^{+}$, is isomorphic to a tensor product of modules of the form $\mathcal{V}\left(\boldsymbol{\pi}_{\lambda, a}\right)$ with distinct $a$.

5.3. The assignment $\varpi_{i, a} \rightarrow \chi_{i, a}$ extends to a surjective map of monoids $\mathcal{P}^{+} \rightarrow \Xi$. Denote by $\chi_{\boldsymbol{\pi}}$ the image of $\boldsymbol{\pi} \in \mathcal{P}^{+}$under this map. Given $\chi \in \Xi$, let $\mathcal{F}_{\chi}$ be the full subcategory of $\mathcal{F}$ whose objects have the following property: $\mathcal{V}(\boldsymbol{\pi})$ is an irreducible constituent of $V$ in $\mathcal{F}_{\chi}$ only if $\chi=\chi_{\boldsymbol{\pi}}$. The following result was proved in [5].

Theorem 3. We have $\mathcal{F}=\bigoplus_{\chi \in \Xi} \mathcal{F}_{\chi}$. Moreover the $\mathcal{F}_{\chi}$ are the blocks in $\mathcal{F}$.

5.4. Define a functor $L: \mathcal{F} \rightarrow \mathcal{I}^{\text {fin }}$ by $L(V)=V \otimes \mathbf{C}\left[t, t^{-1}\right]$ with the $L^{e}(\mathfrak{g})$-module structure given by:

$$
\left(x \otimes t^{k}\right)\left(v \otimes t^{n}\right)=\left(x \otimes t^{k}\right) v \otimes t^{k+n}, \quad d\left(v \otimes t^{n}\right)=n v \otimes t^{n}
$$

for all $x \in \mathfrak{g}, v \in V$ and $k, n \in \mathbf{Z}$. Clearly $L$ preserves direct sums and short exact sequences.

It should be noted that the functor $L$ is not essentially surjective. For example, the indecomposable module $L(\mathfrak{g}) \oplus \mathbf{C}$ with the $L^{e}(\mathfrak{g})$-module structure given by

$$
\left(x \otimes t^{k}\right)\left(y \otimes t^{r}, a\right)=\left([x, y] \otimes t^{r+k}, r \delta_{r,-k}(x, y)_{\mathfrak{g}}\right), \quad d\left(y \otimes t^{r}, a\right)=\left(r y \otimes t^{r}, 0\right)
$$

is not isomorphic to $L(V)$ for any $V$ in $\mathcal{F}$.

Given any $\ell$-highest weight $L(\mathfrak{g})$-module generated by an $\ell$-highest weight vector $v$, let $L^{s}(V)$ be the $L^{e}(\mathfrak{g})$-submodule of $L(V)$ generated by $v \otimes t^{s}$. The following result was proved in [7] (see also [3]).

Proposition. Let $\boldsymbol{\pi} \in \mathcal{P}^{+}, \boldsymbol{\pi} \neq \mathbf{1}$.

(i) For $0 \leq s<m(\boldsymbol{\pi})$ the module $L^{s}(\mathcal{V}(\boldsymbol{\pi}))$ is an irreducible $L^{e}(\mathfrak{g})$-submodule of $L(\mathcal{V}(\boldsymbol{\pi}))$ and moreover,

$$
L(\mathcal{V}(\boldsymbol{\pi}))=\bigoplus_{s=0}^{m(\boldsymbol{\pi})-1} L^{s}(\mathcal{V}(\boldsymbol{\pi})) .
$$

Further, as $L(\mathfrak{g})$-modules, we have $L^{s}(\mathcal{V}(\boldsymbol{\pi})) \cong L^{r}(\mathcal{V}(\boldsymbol{\pi}))$ for all $0 \leq s, r<$ $m(\boldsymbol{\pi})$.

(ii) Any irreducible object in $\mathcal{I}^{\text {fin }}$ is isomorphic to $L^{s}(\mathcal{V}(\boldsymbol{\pi}))$ for some $\boldsymbol{\pi} \in \mathcal{P}^{+}$, $\boldsymbol{\pi} \neq \mathbf{1}$ and $0 \leq s<m(\boldsymbol{\pi})$ or to $\mathbf{C}_{r \delta}$ for some $r \in \mathbf{Z}$.

(iii) As $L^{e}(\mathfrak{g})$-modules $L^{s}(\mathcal{V}(\boldsymbol{\pi})) \cong L^{r}\left(\mathcal{V}\left(\boldsymbol{\pi}^{\prime}\right)\right)$ if and only if $\boldsymbol{\pi}^{\prime}(u)=\boldsymbol{\pi}($ au $)$ for some $a \in \mathbf{C}^{\times}$and $r=s(\bmod m(\boldsymbol{\pi}))$.

5.5. Motivated by the preceding result, we define an action of the group $\mathbf{C}^{\times}$on $\mathcal{P}^{+}$by $(a \cdot \boldsymbol{\pi})(u)=\boldsymbol{\pi}(a u)$ for all $a \in \mathbf{C}^{\times}, \boldsymbol{\pi} \in \mathcal{P}^{+}$and we define $\overline{\mathcal{P}^{+}}$and $\overline{\boldsymbol{\pi}}$ in the obvious way. It is easily checked that the surjective map of monoids $\mathcal{P}^{+} \rightarrow \Xi$ described in Section 5.3 induces a surjective map $\overline{\mathcal{P}^{+}} \rightarrow \Xi$. Denote by $\overline{\chi_{\pi}}$ the image under this map of the element $\overline{\boldsymbol{\pi}}$.

Given $\bar{\chi} \in \bar{\Xi}$ with $\bar{\chi} \neq \overline{0}$, let $\mathcal{I}_{\bar{\chi}}^{\text {fin }}$ be the full subcategory of $\mathcal{I}^{\text {fin }}$ consisting of $L^{e}(\mathfrak{g})$-modules $V \in \mathrm{Ob} \mathcal{I}^{\text {fin }}$ satisfying: $L^{s}(\mathcal{V}(\boldsymbol{\pi}))$ is an irreducible constituent of $V$ only if $\bar{\chi}=\overline{\chi_{\boldsymbol{\pi}}}$. We say that $V$ is in $\mathcal{I}_{\overline{0}}^{\text {fin }}$ if all its simple constituents are either of the form $\mathbf{C}_{r \delta}, r \in \mathbf{Z}$, or of the form $L^{s}(\mathcal{V}(\boldsymbol{\pi}))$ with $\chi_{\boldsymbol{\pi}}=0$. 
Proposition. (i) If $V \in \mathrm{Ob} \mathcal{F}_{\chi}$, then $L(V) \in \mathrm{Ob} \mathcal{I}_{\bar{\chi}}^{\text {fin }}$.

(ii) Suppose that $V$ is an $\ell$-highest weight quotient of $\mathcal{W}(\boldsymbol{\pi})$ for some $\boldsymbol{\pi} \in \mathcal{P}^{+}$and let $v$ be the canonical image of $w_{\boldsymbol{\pi}}$ in $V$. For $0 \leq s<m(\boldsymbol{\pi})$ the submodule $L^{s}(V)=\mathbf{U}\left(L^{e}(\mathfrak{g})\right)\left(v \otimes t^{s}\right) \subset L(V)$ is an $\ell$-highest weight $L^{e}(\mathfrak{g})$-module and

$$
L(V)=\bigoplus_{s=0}^{m(\boldsymbol{\pi})-1} L^{s}(V) .
$$

Proof. Since $V$ is finite dimensional, it has a Jordan-Hölder series $V=V_{N} \supsetneq \cdots \supsetneq$ $V_{0}=0$. By applying the functor $L$ we obtain a filtration $L(V)=L\left(V_{N}\right) \supsetneq \cdots \supsetneq$ $L\left(V_{0}\right)=0$. Observe that $L\left(V_{i}\right) / L\left(V_{i-1}\right) \cong L\left(V_{i} / V_{i-1}\right)$ and thus is either simple or completely reducible. In the second case, it is a finite direct sum of simple objects unless $V_{i} / V_{i-1} \cong \mathbf{C}$ in which case $L\left(V_{i} / V_{i-1}\right) \cong \bigoplus_{r \in \mathbf{Z}} \mathbf{C}_{r \delta}$. Therefore, refining and dropping terms if necessary we can construct a pseudo-Jordan-Hölder series for $L(V)$ out of Jordan-Hölder series for $V$. The statement follows since the pseudo-Jordan-Hölder series is unique up to equivalence defined in Section 4.4.

It suffices to prove (ii) when $V=\mathcal{W}(\boldsymbol{\pi})$ and when $m=m(\boldsymbol{\pi})>1$. Clearly,

$$
L(\mathcal{W}(\boldsymbol{\pi}))=\sum_{s \in \mathbf{Z}} L^{s}(\mathcal{W}(\boldsymbol{\pi})), \quad L^{s}(\mathcal{W}(\boldsymbol{\pi}))=L^{r}(\mathcal{W}(\boldsymbol{\pi})) \quad \text { if } s=r \quad(\bmod m) .
$$

Define $\widehat{\eta}_{m} \in \operatorname{End}_{\mathbf{C}} L(\mathcal{W}(\boldsymbol{\pi}))$ by $\widehat{\eta}_{m}\left(w \otimes t^{r}\right)=\zeta_{m}^{r} \eta_{m}(w) \otimes t^{r}$, where $\zeta_{m}$ is an $m^{t h}$ primitive root of unity and $\eta_{m}$ is a map from Corollary 5.2 (cf. [3, 2.6]). It is easy to check that $\widehat{\eta}_{m} \in \operatorname{End}_{L^{e}(\mathfrak{g})} L(\mathcal{W}(\boldsymbol{\pi}))$ and since $\widehat{\eta}_{m}$ is clearly of order $m$, it defines a representation of $\mathbf{Z} / m \mathbf{Z}$ on $L(\mathcal{W}(\boldsymbol{\pi}))$. It follows that $L(\mathcal{W}(\boldsymbol{\pi}))$ is a direct sum of $\mathbf{Z} / m \mathbf{Z}$-isotypical components corresponding to $m$ distinct irreducible characters of the finite abelian group $\mathbf{Z} / m \mathbf{Z}$. It remains to observe that $\mathbf{Z} / m \mathbf{Z}$ acts by its irreducible character corresponding to $\zeta_{m}^{s}$ on $L^{s}(\mathcal{W}(\boldsymbol{\pi}))$ and hence $L^{s}(\mathcal{W}(\boldsymbol{\pi}))$ is contained in a $\mathbf{Z} / m \mathbf{Z}$-isotypical component of $L(\mathcal{W}(\boldsymbol{\pi}))$.

5.6. For any object $V$ in $\mathcal{I}^{\text {fin }}$, let $V^{\#}=\bigoplus_{\mu \in P_{e}} V_{\mu}^{*} \subset V^{*}$ be the graded dual of $V$. Then $V^{\#}$ is an $L^{e}(\mathfrak{g})$-submodule of $V^{*}$ and is in $\mathcal{I}^{\text {fin }}$, and the functor sending $V$ to $V^{\#}$ is exact and contravariant. It is easy to see that $L(\mathcal{V}(\boldsymbol{\pi}))^{\#} \cong L\left(\mathcal{V}\left(\boldsymbol{\pi}^{*}\right)\right)$, with $\boldsymbol{\pi}^{*} \in \mathcal{P}^{+}$satisfying $\lambda_{\boldsymbol{\pi}^{*}}=-w_{\mathrm{o}} \lambda_{\boldsymbol{\pi}}$ where $w_{\circ}$ is the longest element of $W$. In particular, if $\boldsymbol{\pi}_{j} \in \mathcal{P}^{+}, j=1,2$, and $\lambda_{\boldsymbol{\pi}_{1}}-\lambda_{\boldsymbol{\pi}_{2}} \in Q^{+} \backslash\{0\}$, then $\lambda_{\boldsymbol{\pi}_{2}^{*}}-\lambda_{\boldsymbol{\pi}_{1}^{*}} \notin Q^{+}$.

5.7 .

Proposition. Let $a \in \mathbf{C}^{\times}$and let $\lambda_{j} \in P^{+}, j=1,2$, be such that $\lambda_{1}>\lambda_{2}$. Set $\boldsymbol{\pi}_{j}=\boldsymbol{\pi}_{\lambda_{j}, a}$ and suppose that $\operatorname{Ext}_{\mathcal{F}}^{1}\left(\mathcal{V}\left(\boldsymbol{\pi}_{1}\right), \mathcal{V}\left(\boldsymbol{\pi}_{2}\right)\right) \neq 0$. Assume that $\boldsymbol{\pi} \in \mathcal{P}^{+}$is coprime to $\varpi_{i, a}$ for $i \in I$.

(i) If $m\left(\boldsymbol{\pi}_{1} \boldsymbol{\pi}\right)=1$, then $L\left(\mathcal{V}\left(\boldsymbol{\pi}_{1} \boldsymbol{\pi}\right)\right)$ and $L\left(\mathcal{V}\left(\boldsymbol{\pi}_{2} \boldsymbol{\pi}\right)\right)$ are linked.

(ii) If $m\left(\boldsymbol{\pi}_{1} \boldsymbol{\pi}\right)>1$, then $L\left(\mathcal{V}\left(\boldsymbol{\pi}_{2} \boldsymbol{\pi}\right)\right)$ is simple and is linked to $L^{s}\left(\mathcal{V}\left(\boldsymbol{\pi}_{1} \boldsymbol{\pi}\right)\right)$ for some $0 \leq s<m\left(\boldsymbol{\pi}_{1} \boldsymbol{\pi}\right)$.

Proof. Suppose that we have a non-split short exact sequence of finite-dimensional $L(\mathfrak{g})$-modules

$$
0 \longrightarrow \mathcal{V}\left(\boldsymbol{\pi}_{2}\right) \longrightarrow V \longrightarrow \mathcal{V}\left(\boldsymbol{\pi}_{1}\right) \longrightarrow 0
$$

Since tensoring with $\mathcal{V}(\boldsymbol{\pi})$ is exact and the functor $L$ preserves short exact sequences, we have a short exact sequence of $L^{e}(\mathfrak{g})$-modules,

$$
0 \longrightarrow L\left(\mathcal{V}\left(\boldsymbol{\pi}_{2}\right) \otimes \mathcal{V}(\boldsymbol{\pi})\right) \longrightarrow L(V \otimes \mathcal{V}(\boldsymbol{\pi})) \longrightarrow L\left(\mathcal{V}\left(\boldsymbol{\pi}_{1}\right) \otimes \mathcal{V}(\boldsymbol{\pi})\right) \longrightarrow 0
$$


It is not hard to see (cf. [5]) that the module $V$ is an $\ell$-highest weight module for $L(\mathfrak{g})$ and is a quotient of $\mathcal{W}\left(\boldsymbol{\pi}_{1}\right)$. Moreover, since $\boldsymbol{\pi}$ and $\boldsymbol{\pi}_{j}, j=1,2$, are coprime, it follows from Proposition 5.2 that $V \otimes \mathcal{V}(\boldsymbol{\pi})$ is an $\ell$-highest weight quotient of $\mathcal{W}\left(\boldsymbol{\pi}_{1} \boldsymbol{\pi}\right)$ and also that $\mathcal{V}\left(\boldsymbol{\pi}_{j}\right) \otimes \mathcal{V}(\boldsymbol{\pi}) \cong \mathcal{V}\left(\boldsymbol{\pi}_{j} \boldsymbol{\pi}\right), j=1,2$.

If $m\left(\boldsymbol{\pi}_{1} \boldsymbol{\pi}\right)=1$, then by Proposition 5.5(ii) we see that $L(V \otimes \mathcal{V}(\boldsymbol{\pi}))$ is indecomposable and part (i) is immediate. Otherwise, by Lemma 5.1, $m\left(\boldsymbol{\pi}_{2} \boldsymbol{\pi}\right)=1$ and so $L\left(\mathcal{V}\left(\boldsymbol{\pi}_{2} \boldsymbol{\pi}\right)\right)$ is simple. By Proposition 5.5(ii),

$$
L(V \otimes \mathcal{V}(\boldsymbol{\pi}))=\bigoplus_{s=0}^{m\left(\boldsymbol{\pi}_{1} \boldsymbol{\pi}\right)-1} L^{s}(V \otimes \mathcal{V}(\boldsymbol{\pi}))
$$

and $L\left(\mathcal{V}\left(\boldsymbol{\pi}_{1} \boldsymbol{\pi}\right)\right)=\bigoplus_{s=0}^{m\left(\boldsymbol{\pi}_{1} \boldsymbol{\pi}\right)-1} L^{s}\left(\mathcal{V}\left(\boldsymbol{\pi}_{1} \boldsymbol{\pi}\right)\right)$. It follows from (5.1) that the sequence

$$
0 \longrightarrow L\left(\mathcal{V}\left(\boldsymbol{\pi}_{2} \boldsymbol{\pi}\right)\right) \longrightarrow L^{s}(V \otimes \mathcal{V}(\boldsymbol{\pi})) \longrightarrow L^{s}\left(\mathcal{V}\left(\boldsymbol{\pi}_{1} \boldsymbol{\pi}\right)\right) \longrightarrow 0
$$

is exact for some $0 \leq s<m\left(\boldsymbol{\pi}_{1} \boldsymbol{\pi}\right)$. Part (ii) follows since $L^{s}(V \otimes \mathcal{V}(\boldsymbol{\pi}))$ is $\ell$-highest weight and hence indecomposable.

Corollary. Let $\boldsymbol{\pi} \in \mathcal{P}^{+}$and assume that $m(\boldsymbol{\pi})>1$. Let $a \in \mathbf{C}^{\times}$be such that $\varpi_{i, a}$, $i \in I$, is coprime to $\boldsymbol{\pi}$. Then $L\left(\mathcal{V}\left(\boldsymbol{\pi} \boldsymbol{\pi}_{\theta, a}\right)\right) \sim L(\mathcal{V}(\boldsymbol{\pi}))$. In particular, the modules $L^{s}(\mathcal{V}(\boldsymbol{\pi}))$ and $L^{r}(\mathcal{V}(\boldsymbol{\pi}))$ are linked for any $0 \leq s, r<m(\boldsymbol{\pi})$.

Proof. By Lemma 5.1, $m\left(\boldsymbol{\pi}_{\theta, a} \boldsymbol{\pi}\right)=1$. Since $\operatorname{Ext}_{\mathcal{F}}^{1}\left(\mathcal{V}\left(\boldsymbol{\pi}_{\theta, a}\right), \mathbf{C}\right) \neq 0$ (cf. [5]), the result is immediate from the Proposition.

5.8 .

Proposition. Let $\boldsymbol{\pi}, \boldsymbol{\pi}^{\prime} \in \mathcal{P}^{+}$and suppose that $\chi_{\boldsymbol{\pi}^{\prime}}=0$. Then $L\left(\mathcal{V}\left(\boldsymbol{\pi} \boldsymbol{\pi}^{\prime}\right)\right) \sim$ $L(\mathcal{V}(\boldsymbol{\pi}))$.

Proof. Clearly it is sufficient to prove the statement for $\boldsymbol{\pi}^{\prime}=\boldsymbol{\pi}_{\beta, a}$ where $\beta \in$ $P^{+} \cap Q \subset Q^{+}$.

Suppose first that $\boldsymbol{\pi}_{\beta, a}$ is co-prime with $\boldsymbol{\pi}$. Since $\lambda \in P^{+} \cap Q^{+}$, by [5, Proposition 1.2] there exists a sequence $\gamma_{r} \in P^{+} \cap Q^{+}, r=0, \ldots, N$, such that $\gamma_{0}=\beta$, $\gamma_{N}=0$ and $\operatorname{Ext}_{\mathcal{F}}^{1}\left(\mathcal{V}\left(\boldsymbol{\pi}_{\gamma_{r}, a}\right), \mathcal{V}\left(\boldsymbol{\pi}_{\gamma_{r+1}, a}\right)\right) \neq 0$. It then follows from Proposition 5.7 and its Corollary that the module $L\left(\mathcal{V}\left(\boldsymbol{\pi}_{\gamma_{r}, a} \boldsymbol{\pi}\right)\right)$ is linked to $L\left(\mathcal{V}\left(\boldsymbol{\pi}_{\gamma_{s}, a} \boldsymbol{\pi}\right)\right)$ for all $0 \leq r, s \leq N$, which implies the assertion.

If $\boldsymbol{\pi}_{\beta, a}$ is not co-prime with $\boldsymbol{\pi}$, then we can write $\boldsymbol{\pi}=\boldsymbol{\pi}_{\mu, a} \boldsymbol{\pi}_{1}$ where $\boldsymbol{\pi}_{1}$ is coprime with $\varpi_{i, a}, i \in I$. Then $\boldsymbol{\pi}_{\beta, a}=\boldsymbol{\pi}_{\mu+\beta, a} \boldsymbol{\pi}_{1}$. Again, by [5, Proposition 1.2], there exists a sequence $\nu_{r} \in P^{+}, r=0, \ldots, K$, such that $\nu_{0}=\mu+\beta, \nu_{K}=\mu$ and $\operatorname{Ext}_{\mathcal{F}}^{1}\left(\mathcal{V}\left(\boldsymbol{\pi}_{\nu_{r}, a}\right), \mathcal{V}\left(\boldsymbol{\pi}_{\nu_{r+1}, a}\right)\right) \neq 0$. Then it follows from Proposition 5.7 and its Corollary that $L\left(\mathcal{V}\left(\boldsymbol{\pi}_{\nu_{r}, a} \boldsymbol{\pi}_{1}\right)\right) \sim L\left(\mathcal{V}\left(\boldsymbol{\pi}_{\nu_{s}, a} \boldsymbol{\pi}_{1}\right)\right)$ for all $0 \leq r, s \leq K$.

Corollary. Let $\boldsymbol{\pi}_{j} \in \mathcal{P}^{+}, j=1,2$, be such that $\bar{\chi}_{\boldsymbol{\pi}_{1}}=\bar{\chi}_{\boldsymbol{\pi}_{2}}$. Then $L\left(\mathcal{V}\left(\boldsymbol{\pi}_{1}\right)\right) \sim$ $L\left(\mathcal{V}\left(\boldsymbol{\pi}_{2}\right)\right)$.

Proof. Suppose first that $\bar{\chi}_{\boldsymbol{\pi}_{j}}=0, j=1,2$. Then $\chi_{\boldsymbol{\pi}_{j}}=0$ and it follows from the Proposition that the $L\left(\mathcal{V}\left(\boldsymbol{\pi}_{j}\right)\right), j=1,2$, are linked to $L(\mathbf{C})$ and hence are linked.

Suppose that $\overline{\chi_{\boldsymbol{\pi}_{j}}} \neq 0$. We may assume, without loss of generality, that $\chi_{\boldsymbol{\pi}_{1}}=$ $\chi_{\boldsymbol{\pi}_{2}}$. By the Proposition, we may assume that $\boldsymbol{\pi}_{j}=\prod_{r=1}^{k} \boldsymbol{\pi}_{\lambda_{j, r}, a_{r}}$ such that $\lambda_{1, r}-$ $\lambda_{2, r}=\beta_{r}^{+}-\beta_{r}^{-}, \beta_{r}^{ \pm} \in Q^{+}$. Applying the Proposition again we conclude that $L\left(\mathcal{V}\left(\boldsymbol{\pi}_{2}\right)\right) \sim L\left(\mathcal{V}\left(\boldsymbol{\pi}_{2} \prod_{r=1}^{k} \boldsymbol{\pi}_{\beta_{r}^{+}, a_{r}}\right)\right)=L\left(\mathcal{V}\left(\boldsymbol{\pi}_{1} \prod_{r=1}^{k} \boldsymbol{\pi}_{\beta_{r}^{-}, a_{r}}\right)\right) \sim L\left(\mathcal{V}\left(\boldsymbol{\pi}_{1}\right)\right)$. 


\section{Characters of indecomposable objects in $\mathcal{I}^{\text {fin }}$}

6.1. In order to complete the proof of Theorem 2, it remains to establish the following Proposition.

Proposition. Let $V$ be an indecomposable object in $\mathcal{I}^{\text {fin }}$. Then $V$ is an object in $\mathcal{I}_{\bar{\chi}}^{\text {fin }}$ for some $\chi \in \Xi$.

In order to prove this Proposition we will need to establish some properties of $\ell$-highest weight $L^{e}(\mathfrak{g})$-modules.

6.2. Given $\lambda \in P_{e}^{+}$, let $W^{e}(\lambda)$ be the left $L^{e}(\mathfrak{g})$-module generated by an element $w_{\lambda}$ subject to the relations

$$
L\left(\mathfrak{n}^{+}\right) w_{\lambda}=0, \quad h w_{\lambda}=\lambda(h) w_{\lambda}, \quad\left(x_{\alpha}^{-} \otimes 1\right)^{\lambda\left(\alpha^{\vee}\right)+1} w_{\lambda}=0,
$$

for all $\alpha \in R^{+}$and $h \in \mathfrak{h}_{e}$. Clearly, right multiplication by elements of $\mathbf{U}(L(\mathfrak{h}))$ defines a structure of a right $\mathbf{U}(L(\mathfrak{h}))$-module on $W^{e}(\lambda)$. The following can be found in [8].

Proposition. (i) Let $J_{\lambda}=\operatorname{Ann}_{\mathbf{U}(L(\mathfrak{h}))} w_{\lambda}$. Given $\boldsymbol{\pi} \in \mathcal{P}^{+}$with $\lambda_{\boldsymbol{\pi}}=\lambda$, there exists a maximal ideal $J_{\boldsymbol{\pi}} \supset J_{\lambda}$ in $\mathbf{U}(L(\mathfrak{h}))$ such that one has an isomorphism of $L(\mathfrak{g})$-modules

$$
\mathcal{W}(\boldsymbol{\pi}) \cong W^{e}(\lambda) \otimes_{\mathbf{U}(L(\mathfrak{h}))} \mathbf{U}(L(\mathfrak{h})) / J_{\boldsymbol{\pi}} .
$$

Conversely if $J$ is any maximal ideal in $\mathbf{U}(L(\mathfrak{h})) / J_{\lambda}$, then $J=J_{\boldsymbol{\pi}}$ for some $\boldsymbol{\pi} \in \mathcal{P}^{+}$and

$$
\mathcal{W}(\boldsymbol{\pi}) \cong W^{e}(\lambda) \otimes_{\mathbf{U}(L(\mathfrak{h}))} \mathbf{U}(L(\mathfrak{h})) / J .
$$

6.3. As explained in the Introduction, the next theorem follows from [1], [13, 12]. It was also proved, by different methods, in [8] for $\mathfrak{g}=\mathfrak{s l}_{2}$, in [4] for $\mathfrak{g}=\mathfrak{s l}_{n}, n \geq 3$, and in $[10]$ for $\mathfrak{g}$ of all simply laced types.

Theorem 4. Let $\lambda \in P^{+}$. Then there exists $N_{\lambda}>0$ such that $\operatorname{dim} \mathcal{W}(\boldsymbol{\pi})=N_{\lambda}$ for all $\boldsymbol{\pi} \in \mathcal{P}^{+}$satisfying $\lambda_{\boldsymbol{\pi}}=\lambda$.

Corollary. As a $\mathbf{U}(L(\mathfrak{h})) / J_{\lambda}$-module, $W^{e}(\lambda)$ is free of rank $N_{\lambda}$.

Proof. Let $S_{\lambda}=\mathbf{U}(L(\mathfrak{h})) / J_{\lambda}$. Then any maximal ideal in $S_{\lambda}$ is of the form $J_{\boldsymbol{\pi}}$ for some $\boldsymbol{\pi} \in \mathcal{P}^{+}$with $\lambda_{\boldsymbol{\pi}}=\lambda$. Since $\mathcal{W}(\boldsymbol{\pi}) \cong W^{e}(\lambda) \otimes_{S_{\lambda}} S_{\lambda} / J_{\boldsymbol{\pi}}$, it follows from the Theorem that $\operatorname{dim} W^{e}(\lambda) \otimes_{S_{\lambda}} S_{\lambda} / J=N_{\lambda}$ for any maximal ideal $J$ in $S_{\lambda}$. The statement follows by Nakayama's Lemma.

6.4. Let $K_{\boldsymbol{\pi}}$ be the kernel of the homomorphism $\psi_{\boldsymbol{\pi}}: \mathbf{U}(L(\mathfrak{h})) \rightarrow \mathbf{C}\left[t^{ \pm 1}\right]$ of $\mathbf{Z}$ graded algebras defined by $\psi_{\boldsymbol{\pi}}\left(\Lambda_{i, \pm s}\right)=\pi_{i, s}^{ \pm} t^{ \pm s}$. Then $K_{\boldsymbol{\pi}} \supset J_{\lambda_{\boldsymbol{\pi}}}$ and we set

$$
W^{e}(\boldsymbol{\pi})=W^{e}\left(\lambda_{\boldsymbol{\pi}}\right) \otimes_{\mathbf{U}(L(\mathfrak{h}))} \mathbf{U}(L(\mathfrak{h})) / K_{\boldsymbol{\pi}} .
$$

It is clear that any $\ell$-highest weight $L^{e}(\mathfrak{g})$-module is a quotient of $W^{e}(\boldsymbol{\pi}) \otimes \mathbf{C}_{s \delta}$ for some $\boldsymbol{\pi} \in \mathcal{P}^{+}, s \in \mathbf{Z}$.

Proposition. The module $W^{e}(\boldsymbol{\pi})$ is a free module over $\mathbf{U}(L(\mathfrak{h})) / K_{\boldsymbol{\pi}}$ of rank $\operatorname{dim} \mathcal{W}(\boldsymbol{\pi})$. In particular $W^{e}(\boldsymbol{\pi}) \cong L^{0}(\mathcal{W}(\boldsymbol{\pi}))$ as $L^{e}(\mathfrak{g})$-modules. 
Proof. The first statement is a standard consequence of the Corollary of Theorem 4. For the second, let $m=m(\boldsymbol{\pi})$ and let $\eta_{m} \in \operatorname{End}_{\mathbf{C}} \mathcal{W}(\boldsymbol{\pi})$ be the map from Corollary 5.2. By the proof of Proposition 5.5(ii), $L^{0}(\mathcal{W}(\boldsymbol{\pi}))$ is spanned by elements $w \otimes t^{s}, s \in \mathbf{Z}$, where $w \in \mathcal{W}(\boldsymbol{\pi})$ satisfies $\eta_{m}(w)=\zeta_{m}^{-s} w, \zeta_{m}$ being an $m^{t h}$ complex primitive root of unity. Since $\pi_{i, s}^{ \pm}=0$ unless $m$ divides $s$, it follows that the formula

$$
\left(w \otimes t^{r}\right) \Lambda_{i, \pm s}=\pi_{i, s}^{ \pm}\left(w \otimes t^{r \pm s}\right)
$$

defines a right action of $\mathbf{U}(L(\mathfrak{h}))$ on $L^{0}(\mathcal{W}(\boldsymbol{\pi}))$ which commutes with the left $L(\mathfrak{g})$ module action. It follows that $L^{0}(\mathcal{W}(\boldsymbol{\pi}))$ is a free module for $\mathbf{U}(L(\mathfrak{h})) / K_{\boldsymbol{\pi}}$ of rank $\operatorname{dim} \mathcal{W}(\boldsymbol{\pi})$.

The Proposition follows by noticing that $L^{0}(\mathcal{W}(\boldsymbol{\pi}))$ is an $L^{e}(\mathfrak{g})$-module quotient of $W^{e}(\boldsymbol{\pi})$, that the quotient map is also a map of right $\mathbf{U}(L(\mathfrak{h}))$-modules and that $L^{0}(\mathcal{W}(\boldsymbol{\pi}))$ and $W^{e}(\boldsymbol{\pi})$ are free $\mathbf{U}(L(\mathfrak{h})) / K_{\boldsymbol{\pi}}$-modules of the same rank.

Corollary. Let $V$ be an $\ell$-highest weight $L^{e}(\mathfrak{g})$-module. Then $V$ is a quotient of $L^{s}(\mathcal{W}(\boldsymbol{\pi}))$ for some $\boldsymbol{\pi} \in \mathcal{P}^{+}, 0 \leq s<m(\boldsymbol{\pi})$.

6.5. The crucial point in the proof of Proposition 6.1 is the following result.

Proposition. Suppose that $V_{i}, i=1,2$, are simple objects in $\mathcal{I}_{\bar{\chi}_{i}}^{\text {fin }}$ respectively, with $\bar{\chi}_{1} \neq \bar{\chi}_{2}$. Then $\operatorname{Ext}_{\mathcal{I}_{f i n}}^{1}\left(V_{1}, V_{2}\right)=0$.

Proof. Suppose that there exists a non-split short exact sequence of $L^{e}(\mathfrak{g})$-modules

$$
0 \longrightarrow V_{2} \stackrel{\phi}{\longrightarrow} M \stackrel{\xi}{\longrightarrow} V_{1} \longrightarrow 0 .
$$

Since $\mathbf{C}_{r \delta} \in \mathrm{ObI}_{\overline{0}}^{\text {fin }}$ for all $r \in \mathbf{Z}$, we may assume that at least one of $V_{1}$ or $V_{2}$ is not isomorphic to $\mathbf{C}_{r \delta}$ for $r \in \mathbf{Z}$. By passing to graded duals if necessary (cf. Section 5.6), we can assume that $V_{1} \cong L^{s}\left(\mathcal{V}\left(\boldsymbol{\pi}_{1}\right)\right)$ for some $\boldsymbol{\pi}_{1} \in \mathcal{P}^{+}$with $\boldsymbol{\pi}_{1} \neq \mathbf{1}, 0 \leq s<m\left(\boldsymbol{\pi}_{1}\right)$, and also that if $V_{2} \cong L^{r}\left(\mathcal{V}\left(\boldsymbol{\pi}_{2}\right)\right), 0 \leq r<m\left(\boldsymbol{\pi}_{2}\right)$, then $\lambda_{\boldsymbol{\pi}_{2}}-\lambda_{\boldsymbol{\pi}_{1}} \notin Q^{+}$. Since $L^{s}\left(\mathcal{V}\left(\boldsymbol{\pi}_{1}\right)\right) \cong L^{0}\left(\mathcal{V}\left(\boldsymbol{\pi}_{1}\right)\right) \otimes \mathbf{C}_{s \delta}$, we further assume by tensoring the above short exact sequence, if necessary, with $\mathbf{C}_{-s \delta}$ that $s=0$. Choose $m_{\boldsymbol{\pi}_{1}} \in M$ such that $\xi\left(m_{\boldsymbol{\pi}_{1}}\right)=v_{\boldsymbol{\pi}_{1}} \otimes 1$. Since the sequence is not split and $\lambda_{\boldsymbol{\pi}_{2}} \ngtr \lambda_{\boldsymbol{\pi}_{1}}$, we have

$$
L\left(\mathfrak{n}^{+}\right) m_{\boldsymbol{\pi}_{1}}=0, \quad M=\mathbf{U}\left(L^{e}(\mathfrak{g})\right) m_{\boldsymbol{\pi}_{1}} .
$$

In particular, if we denote by $M_{0}$ the $\mathbf{U}\left(L^{e}(\mathfrak{h})\right)$-submodule of $M$ generated by $m_{\boldsymbol{\pi}_{1}}$, then we have

$$
M_{0}=\bigoplus_{r \in \mathbf{Z}} M_{\lambda_{\pi_{1}}+r \delta} \cong \bigoplus_{r \in \mathbf{Z}}\left(\left(V_{1}\right)_{\lambda_{\pi_{1}}+r \delta} \oplus\left(V_{2}\right)_{\lambda_{\pi_{1}}+r \delta}\right)
$$

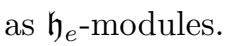

If $\operatorname{dim} M_{\lambda_{\pi_{1}}+r \delta} \leq 1$ (in particular, if $\lambda_{\boldsymbol{\pi}_{1}} \neq \lambda_{\boldsymbol{\pi}_{2}}$ ), then $M$ is an $\ell$-highest weight module for $L^{e}(\mathfrak{g})$. Then by Proposition $6.4 M$ is a quotient of $L^{s}\left(\mathcal{W}\left(\boldsymbol{\pi}_{1}\right)\right)$. Since Proposition 5.4 implies that $L^{s}\left(\mathcal{W}\left(\pi_{1}\right)\right) \in \mathrm{Ob}_{\bar{\chi}_{\pi_{1}}}^{\text {fin }}$ it follows that $M \in$ Ob $\mathcal{I}_{\bar{\chi} \boldsymbol{\pi}_{1}}^{\text {fin }}$. That is impossible if $\bar{\chi}_{\boldsymbol{\pi}_{2}} \neq \bar{\chi}_{\boldsymbol{\pi}_{1}}$ and we obtain a contradiction.

If $\lambda_{\boldsymbol{\pi}_{1}}=\lambda_{\boldsymbol{\pi}_{2}}=\lambda$ and $\operatorname{dim} M_{\lambda+p_{0} \delta}=2$ for some $p_{0} \in \mathbf{Z}$, then $\operatorname{dim} M_{\lambda+p \delta}=2$ for all $p=p_{0}(\bmod k)$ where $k=\operatorname{lcm}\left(m\left(\boldsymbol{\pi}_{1}\right), m\left(\boldsymbol{\pi}_{2}\right)\right)$. Fix $m_{\boldsymbol{\pi}_{1}, l} \in M_{\lambda+\left(p_{0}+k l\right) \delta}$ such that $\xi\left(m_{\boldsymbol{\pi}_{1}, l}\right)=v_{\boldsymbol{\pi}_{1}} \otimes t^{p_{0}+k l}$ and set $m_{\boldsymbol{\pi}_{2}, l}=\phi\left(v_{\boldsymbol{\pi}_{2}} \otimes t^{p_{0}+k l}\right) \in M_{\lambda+\left(p_{0}+k l\right) \delta}$. Then $\left\{m_{\boldsymbol{\pi}_{1}, l}, m_{\boldsymbol{\pi}_{2}, l}\right\}$ is a basis of $M_{\lambda+\left(p_{0}+k l\right) \delta}$. Let $\bar{\psi}_{\boldsymbol{\pi}}$ denote the composition 
of $\psi_{\boldsymbol{\pi}}$ with the map $\mathbf{C}\left[t^{ \pm 1}\right] \rightarrow \mathbf{C}, t \mapsto 1$. Since $\bar{\chi}_{\boldsymbol{\pi}_{1}} \neq \bar{\chi}_{\boldsymbol{\pi}_{2}}$ there exists $l, s \in \mathbf{Z}$ and $x \in \mathbf{U}(L(\mathfrak{h}))_{k s}$ such that $\bar{\psi}_{\boldsymbol{\pi}_{1}}(x) \neq \bar{\psi}_{\boldsymbol{\pi}_{2}}(x)$ and

$$
x m_{\boldsymbol{\pi}_{1}, l}=\bar{\psi}_{\boldsymbol{\pi}_{1}}(x) m_{\boldsymbol{\pi}_{1}, l+s}+c m_{\boldsymbol{\pi}_{2}, l+s}, \quad x m_{\boldsymbol{\pi}_{2}, l}=\bar{\psi}_{\boldsymbol{\pi}_{2}}(x) m_{\boldsymbol{\pi}_{2}, l+s},
$$

where $c \in \mathbf{C}$. Let $\gamma: M_{\lambda+\left(p_{0}+(l+s) k\right) \delta} \rightarrow M_{\lambda+\left(p_{0}+k l\right) \delta}$ be the isomorphism of vector spaces defined by $\gamma\left(m_{\boldsymbol{\pi}_{j}, l+s}\right)=m_{\boldsymbol{\pi}_{j}, l}, j=1,2$. Then the matrix of $\gamma \circ x$ in the basis $\left\{m_{\boldsymbol{\pi}_{1}, l}, m_{\boldsymbol{\pi}_{2}, l}\right\}$ is upper triangular with distinct diagonal entries and so $\gamma \circ x$ has two one-dimensional eigenspaces. Obviously, $m_{\boldsymbol{\pi}_{2}, l}$ is an eigenvector of $\gamma \circ x$ corresponding to the eigenvalue $\bar{\psi}_{\boldsymbol{\pi}_{2}}(x)$. On the other hand, since $m_{\boldsymbol{\pi}_{2}, l} \in$ $\mathbf{U}\left(L^{e}(\mathfrak{h})\right) m_{\boldsymbol{\pi}_{1}, l}$, it follows that $m_{\boldsymbol{\pi}_{2}, l}=y m_{\boldsymbol{\pi}_{1}, l}$ for some $y \in \mathbf{U}\left(L^{e}(\mathfrak{h})\right)_{0}$. Now

$$
y \gamma\left(x m_{\boldsymbol{\pi}_{1}, l}\right)=\gamma\left(x y m_{\boldsymbol{\pi}_{1}, l}\right),
$$

and hence $m_{\boldsymbol{\pi}_{2}, l}$ is also an eigenvector of $\gamma \circ x$ corresponding to the eigenvalue $\bar{\psi}_{\boldsymbol{\pi}_{1}}(x)$, which is a contradiction.

Corollary. Suppose that $V_{i} \in \mathrm{Ob} \mathcal{I}_{\bar{\chi}_{i}}^{\text {fin }}, i=1,2$, with $\bar{\chi}_{1} \neq \bar{\chi}_{2}$. Then $\operatorname{Ext}_{\mathcal{I}^{f i n}}^{1}\left(V_{1}, V_{2}\right)$ $=0$.

Proof. An induction on the pseudo-length (similar to the induction on length used in $[5$, Lemma 5.2] for $\mathcal{F}$ ) yields the Corollary.

6.6. We are now able to prove Proposition 6.1.

Proof. Let $V$ be an indecomposable object in $\mathcal{I}^{\text {fin }}$. We proceed by induction on the pseudo-length of $V$. If $V$ is simple, then it is clear from the definition that $V \in \mathcal{I}_{\bar{\chi}_{\boldsymbol{\pi}}}^{\text {fin }}$ for some $\boldsymbol{\pi} \in \mathcal{P}^{+}$. Otherwise, we have an extension

$$
0 \longrightarrow V_{1} \longrightarrow U \longrightarrow 0
$$

where either $V_{1}=V^{L(\mathfrak{g})}$ or $V_{1} \not \subset V^{L(\mathfrak{g})}$ and is simple. In any case, $V_{1}$ is an object in $\mathcal{I}_{\bar{\chi}}^{\text {fin }}$ for some $\chi \in \Xi$. Using Theorem 1 , we can write

$$
U=\bigoplus_{j=0}^{k} U_{j}
$$

where $U_{0}=\bigoplus_{r \in \mathbf{Z}} \mathbf{C}_{r \delta}^{m_{r}}, m_{r} \geq 0$, and for $j=1, \ldots, k$ the module $U_{j}$ is indecomposable with wt ${ }^{e}\left(U_{j}\right) \not \subset \mathbf{Z} \delta$. Notice that the pseudo-length of $U_{j}, j \geq 1$, is strictly smaller than that of $V$ unless $U$ is indecomposable and $V_{1}=V^{L(\mathfrak{g})}$.

Suppose first that $V_{1} \neq V^{L(\mathfrak{g})}$. Then, by the induction hypothesis, we see that for $j=1, \ldots, k, U_{j}$ is an object in $\mathcal{I}_{\bar{\chi}_{j}}^{\text {fin }}$ for some $\chi_{j} \in \Xi$. Suppose that $\bar{\chi} \neq \bar{\chi}_{j_{0}}$ for some $0 \leq j_{0} \leq k$. Then by Corollary 6.5

$$
\operatorname{Ext}_{\mathcal{I}^{f i n}}^{1}\left(U, V_{1}\right) \cong \bigoplus_{j \neq j_{0}} \operatorname{Ext}_{\mathcal{I}^{f i n}}^{1}\left(U_{j}, V_{1}\right) .
$$

In other words, the exact sequence (6.1) is equivalent to

$$
0 \longrightarrow V_{1} \longrightarrow U_{j_{0}} \oplus V^{\prime} \longrightarrow U_{j_{0}} \oplus \bigoplus_{j \neq j_{0}} U_{j} \longrightarrow 0,
$$

where

$$
0 \longrightarrow V_{1} \longrightarrow V^{\prime} \longrightarrow \bigoplus_{j \neq j_{0}} U_{j} \longrightarrow 0
$$


represents an element of $\bigoplus_{j \neq j_{0}} \operatorname{Ext}_{\mathcal{I}^{f i n}}^{1}\left(U_{j}, V_{1}\right)$. This is a contradiction since $V$ is indecomposable. Finally, suppose that $U$ is indecomposable and $V_{1}=V^{L(\mathfrak{g})}$. Then $U^{L(\mathfrak{g})}=0$ and so we have a short exact sequence

$$
0 \longrightarrow U_{1} \longrightarrow U \longrightarrow U_{2} \longrightarrow 0,
$$

where $U_{1}$ is simple and $U_{1} \not \subset U^{L(\mathfrak{g})}$. Hence the pseudo-length of $U_{2}$ is strictly smaller than that of $U$, and so we can apply the above argument and show that $U$ is an object in $\mathcal{I}_{\bar{\chi}}^{\text {fin }}$ for some $\chi \in \Xi$. If $\bar{\chi} \neq 0$, then $\operatorname{Ext}_{\mathcal{I}^{f i n}}^{1}\left(U, V_{1}\right)=0$ by Corollary 6.5 , and so $V \cong V_{1} \oplus U$ which is a contradiction. Thus, $\bar{\chi}=0$ and $V$ is an object in $\mathrm{Ob} \mathcal{I}_{\overline{0}}^{\text {fin }}$.

\section{LisT OF NOTATION}

\begin{tabular}{|c|c|c|c|}
\hline $\mathfrak{g}, \mathfrak{h}$ & 2.1, p. 2924 & $\mathcal{I}^{\text {fin }}$ & 3.2, p. 2926 \\
\hline$I, \alpha_{i}, \varpi_{i}$ & 2.1, p. 2924 & $V_{\lambda}^{+}$ & 3.3, p. 2927 \\
\hline$R, R^{+}, Q, Q^{+}, P, P^{+}$ & 2.1, p. 2924 & $V^{L(\mathfrak{g})}$ & 3.5, p. 2928 \\
\hline$\theta, \theta_{s}$ & 2.1, p. 2924 & $(\cdot, \cdot)_{\mathfrak{g}}$ & 4, p. 2928 \\
\hline$W$ & 2.1, p. 2924 & $\sim$ & 4.6, p. 2931 \\
\hline$x_{\alpha}^{ \pm}, \alpha^{\vee}$ & 2.1, p. 2924 & $\Xi, \bar{\Xi}$ & 4.7, p. 2931 \\
\hline$\Gamma, \varpi_{\gamma}$ & 2.1, p. 2924 & $\chi_{i, a}$ & 4.7, p. 2931 \\
\hline$V(\lambda), v_{\lambda}$ & 2.2, p. 2925 & $\mathcal{P}^{+}$ & 5.1, p. 2931 \\
\hline $\mathbf{U}(\mathfrak{a})$ & 2.3, p. 2925 & $\varpi_{i, a}, \boldsymbol{\pi}_{\lambda, a}$ & 5.1, p. 2931 \\
\hline$L(\mathfrak{a}), L^{e}(\mathfrak{a}), d$ & 2.3, p. 2925 & $\lambda_{\boldsymbol{\pi}}, m(\boldsymbol{\pi})$ & 5.1, p. 2931 \\
\hline $\mathfrak{h}_{e}$ & 2.4, p. 2925 & $\mathcal{F}$ & 5.2, p. 2932 \\
\hline$\delta$ & 2.4, p. 2925 & $\mathcal{W}(\boldsymbol{\pi}), w_{\boldsymbol{\pi}}, \mathcal{V}(\boldsymbol{\pi}), v_{\boldsymbol{\pi}}$ & 5.2, p. 2932 \\
\hline$P_{e}, P_{e}^{+}$ & 2.4 , p. 2925 & $\tau_{a}$ & 5.2, p. 2932 \\
\hline$\widehat{W}$ & 2.4, p. 2925 & $\chi_{\pi}$ & 5.3, p. 2933 \\
\hline$r_{\lambda}$ & 2.4, p. 2925 & $\mathcal{F}_{\chi}$ & 5.3, p. 2933 \\
\hline$\Lambda_{i}^{ \pm}(u), \Lambda_{i, \pm k}$ & 2.5, p. 2926 & $L$ & 5.4, p. 2933 \\
\hline $\mathcal{I}$ & 3.1 , p. 2926 & $L^{s}(\mathcal{V}(\pi))$ & 5.4, p. 2933 \\
\hline $\mathrm{wt}^{e}(V)$ & 3.1 , p. 2926 & $\mathcal{I}_{\bar{\chi}}^{\text {fin }}$ & 5.5, p. 2933 \\
\hline $\mathbf{C}_{a \delta}$ & 3.2 , p. 2926 & $\#^{\lambda}$ & 5.6, p. 2934 \\
\hline
\end{tabular}

\section{ACKNOWLEDGMENTS}

We are grateful to Hiraku Nakajima for discussions relating to the dimension conjecture.

\section{REFERENCES}

1. J. Beck and H. Nakajima, Crystal bases and two-sided cells of quantum affine algebras, Duke Math. J. 123 (2004), no. 2, 335-402 MR2066942 (2005e:17020)

2. V. Chari, Integrable representations of affine Lie-algebras, Invent. Math. 85 (1986), no. 2, 317-335 MR846931 (88a:17034)

3. V. Chari and J. Greenstein, Quantum loop modules, Represent. Theory 7 (2003), 56-80 (electronic) MR1973367 (2004b:17044)

4. V. Chari and S. Loktev, Weyl, Fusion and Demazure modules for the current algebra of $\mathfrak{s l}_{r+1}$, Adv. Math. 207 (2006), no. 2, 928-960. MR2271991

5. V. Chari and A. A. Moura, Spectral characters of finite-dimensional representations of affine algebras, J. Algebra 279 (2004), no. 2, 820-839 MR2078944 (2005f:17002)

6. Characters and blocks for finite-dimensional representations of quantum affine algebras, Int. Math. Res. Not. 2005, no. 5, 257-298 MR2130797 (2006a:17021) 
7. V. Chari and A. Pressley, New unitary representations of loop groups, Math. Ann. 275 (1986), no. 1, 87-104 MR849057 (88f:17029)

8. _ Weyl modules for classical and quantum affine algebras. Represent. Theory 5 (2001), 191-223 MR1850556 (2002g:17027)

9. P. I. Etingof and A. A. Moura, Elliptic central characters and blocks of finite dimensional representations of quantum affine algebras, Represent. Theory 7 (2003), 346-373 (electronic) MR2017062 (2004j:17017)

10. G. Fourier and P. Littelmann, Weyl modules, Demazure modules, KR-modules, crystals, fusion products and limit constructions, Adv. Math. 211 (2007), no. 2, 566-593 MR2323538

11. J. E. Humphreys, Introduction to Lie algebras and representation theory, Springer, New York, 1978 MR499562 (81b:17007)

12. M. Kashiwara, Crystal bases of modified quantized enveloping algebra, Duke Math. J. 73 (1994), no. 2, 383-413 MR1262212 (95c:17024)

13. On level-zero representations of quantized affine algebras, Duke Math. J. 112 (2002), no. 1, 117-175 MR1890649 (2002m:17013)

14. H. Nakajima, Quiver varieties and finite-dimensional representations of quantum affine algebras, J. Amer. Math. Soc. 14 (2001), no. 1, 145-238 (electronic) MR1808477 (2002i:17023)

15. Extremal weight modules of quantum affine algebras, Adv. Stud. Pure Math. 40 (2004), 343-369 MR2074599 (2005g:17036)

16. S. Eswara Rao, Complete reducibility of integrable modules for the affine Lie (super)algebras, J. Algebra 264 (2003), no. 1, 269-278 MR1980697 (2004b:17016)

Department of Mathematics, University of California, Riverside, California 92521 E-mail address: vyjayanthi.chari@ucr.edu

Department of Mathematics, University of California, Riverside, California 92521

E-mail address: jacob.greenstein@ucr.edu 